\title{
Stereoselective Aminoiodination of Activated Alkynes with Organoiodine(III) Reagents and Amines via Multiple-Site Functionalization: Access to Iodinated Enamines and $N$-Aryl Indoles
}

\author{
Sagar Arepally, Ajoy Chamuah and Duddu S. Sharada*
}

Department of Chemistry, Indian Institute of Technology Hyderabad, Kandi - 502 285, Sangareddy, Telangana,

INDIA, Phone: (040) 2301 7058; Fax: (040) 2301 6032, E-mail: sharada@iith.ac.in

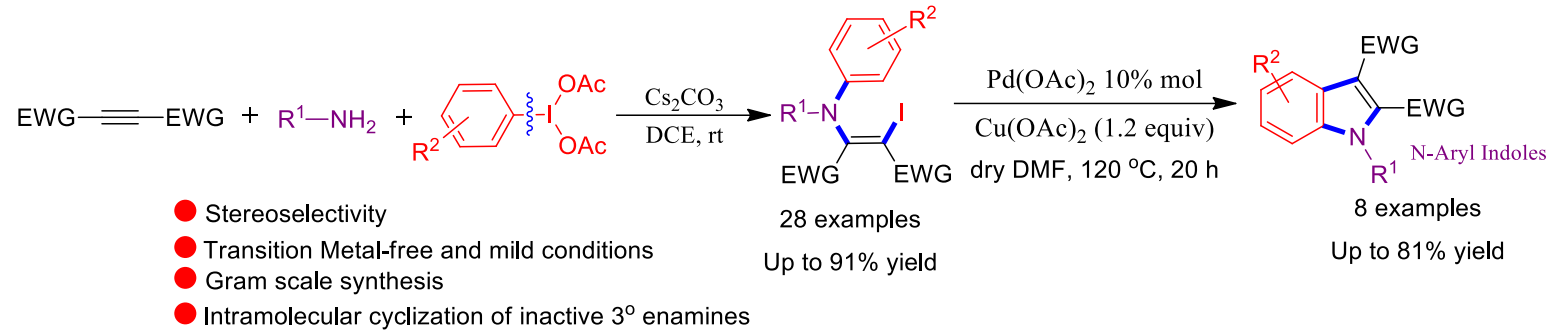

Intramolecular cyclization of inactive $3^{\circ}$ enamines

Abstract: A stereoselective aminoiodination of activated alkynes with $\mathrm{PhI}(\mathrm{OAc})_{2}$ and amines via multiplesite functionalization to afford (Z)diethyl 2-(diphenylamino)-3-iodomaleate derivatives with superior yields has been described. The key feature of this reaction is the incorporation of iodide and aryl group concurrently in the same molecule in a stereoselective manner by employing $\mathrm{PhI}(\mathrm{OAc})_{2}$ as electrophilic reagent as well as iodide and aryl group source. The high stereoselectivity of the reaction can be explained based on the structure of the possible intermediates, the conformations of which controlled by the hydrogen bonding, steric hindrance and electrostatic attractions. This reaction proceeds under mild conditions, providing various dialkyl 2-(diphenylamino)-3-iodomaleates by a single operation starting from activated alkynes. The robustness of our strategy is revealed by making of bis (dialkyl 2-(diphenylamino)-3iodomaleate) derivatives involving formation of four new C-N bonds and two C-I bonds with a single step. The synthesized inactive $3^{\circ}$ enamines (dialkyl 2-(diphenylamino)-3-iodomaleates) could be further transformed into highly substituted indoles via Pd catalyzed C-H and C-I activation under non-acidic conditions. 


\section{Introduction}

Alkyne difunctionalizations have attracted intensive attention in recent years particularly in developing regio and stereoselective reactions to access multifunctional alkene products with broad synthetic and biological applications. ${ }^{1}$ For example the azidativehalo, ${ }^{2}$ sulfonative,${ }^{3}$ aminohalogenative,${ }^{4}$ silylzincative ${ }^{5}$

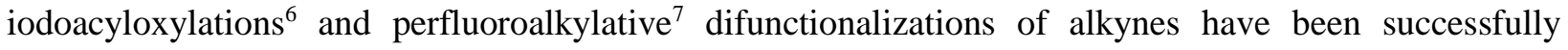
realized for the synthesis of various difunctionalization products. In this context, methods to produce halogenated enamines via aminohalogenation of alkynes are very attractive owing to the utility of these compounds in medicinal chemistry and organic synthesis. ${ }^{8}$ Accordingly, considerable efforts have been made to their syntheses resulting in various synthetic strategies. For example cyclic enaminones, ${ }^{9}$ 2alkynyloxycarbonyl azides/amines/O-propargyl carbamates ${ }^{10}$ have been typically employed as precursors for halogenated enamines and catalyzed by metal or non-metals with various halogen sources.

\section{Scheme 1. Aminohalogenation of Alkynes and Our Strategy}

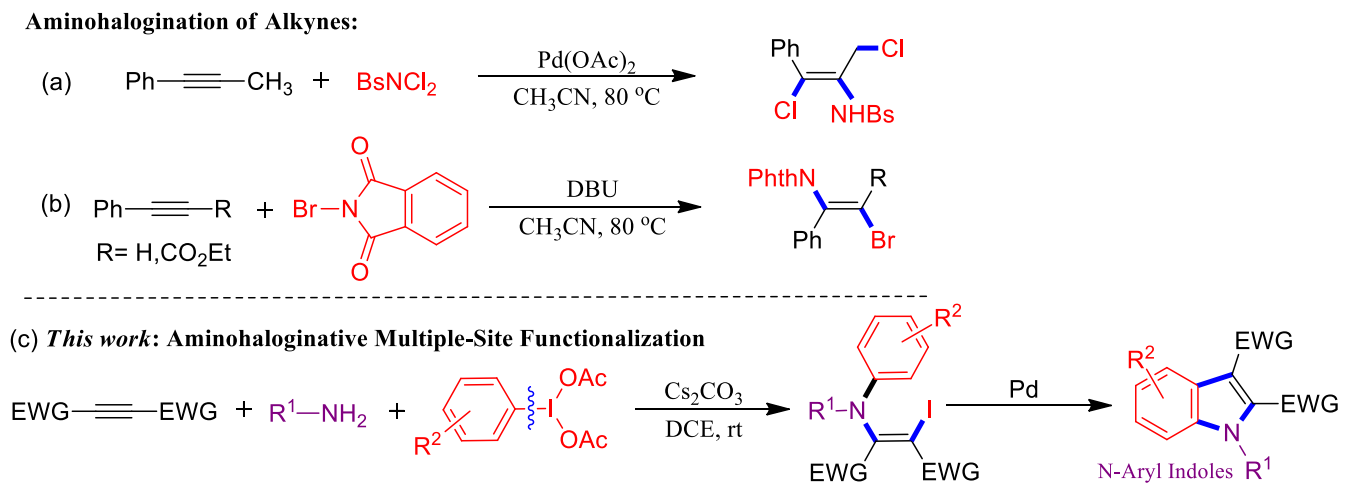

Furthermore, dehydrogenative aminohalogenation of alkenes via Pd catalysis was developed by Jiang and co-workers ${ }^{11}$ for the synthesis of brominated enamines. Later Li et al. ${ }^{12}$ developed Nickel or Diacetoxyiodobenzene promoted halogenation of enamines and enamides. However, examples of alkyne aminohalogenation towards halogenated enamines are scarce (Scheme 1a \&b). ${ }^{13}$ For instance, Headley \& $\mathrm{Li}$ and co-workers demonstrated the aminochlorination of arylalkynes with N,N dichlorobenzenesulfonamide (Scheme 1a). ${ }^{13 a}$ In 2014, Liang and Zhang et al. described the 
aminohalogenation of alkynes with N-haloimides activated by DBU (Scheme 1b). ${ }^{13 \mathrm{~b}}$ Despite this progress, developing the multiple-site functionalization of C-C multiple bonds with reagents as both halogen source as well as promoter in a stereoselective manner remains an intriguing challenge. As part of our ongoing study on multiple functionalization reactions of alkynes and amines using azides as nitrogen source and organoiodine compounds as promoter, ${ }^{14}$ we fancied to synthesize iodinated enamines directly from alkynes and amines by using organoiodine reagents as both iodine and aryl group source.

\section{Scheme 2. Plausible Conformations of Intermediates for Stereoselectivity of the Reaction}

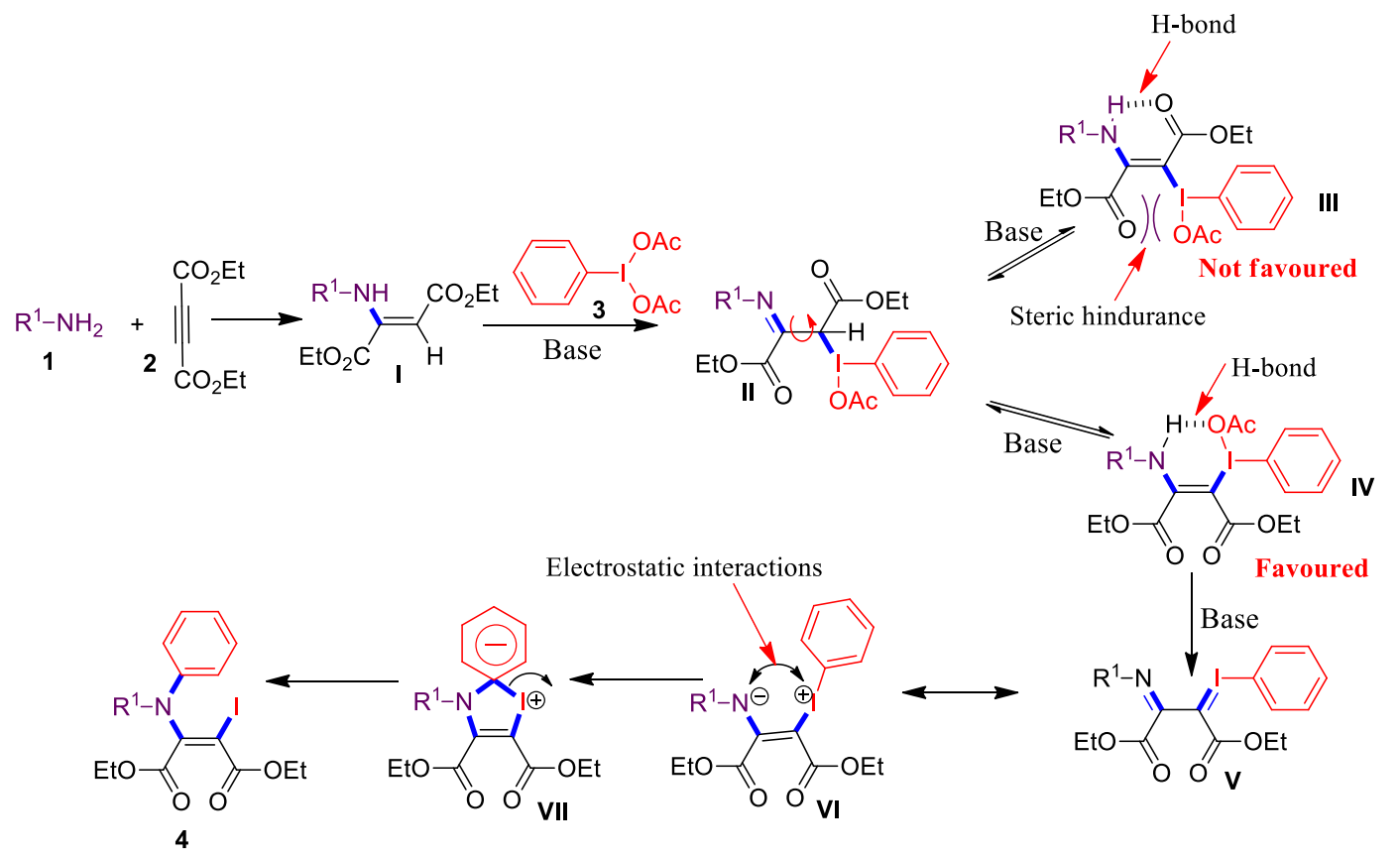

Organoiodine(III) compounds are usually used as oxidants and electrophilic reagents where only one ligand of iodine(III) is removed by the substrate or replacement of both ligands with external nucleophile followed by its decomposition into radicals ${ }^{12,15,16}$ However, sequential removal of two ligands from the iodine(III) reagent by active $\mathrm{C}-\mathrm{H}$ bond of substrate and incorporation of iodobenzene into the same substrate would indubitably make the reactions atom economic, but such organic transformations are not much explored..$^{9}$ On the other hand, compared with known aminohalogenation of alkynes, this multiple-site functionalization would be of great importance to produce synthetically potential halogenated enamines in a stereoselective manner. The high stereoselectivity of the reaction can be explained based on the structure 
of the possible intermediates. The conformations of intermediates are controlled by the hydrogen bonding, steric hindrance and electrostatic attractions as shown in scheme 2. These type of interactions have never been explored for stereoselective alkyne aminohalogenation. Herein we report the first example of a highly stereoselective multiple-site functionalization of activated alkynes with amines and organoiodine(III) (Scheme 1c) as the halo and aryl group source for haloenamines. We have also successfully employed these relatively inactive $3^{\circ}$ enamines in intramolecular cyclizations via $\mathrm{Pd}$ catalyzed $\mathrm{C}-\mathrm{H}$ and C-I activation resulting in highly functionalized indoles.

Table 1. Optimization reaction of Aminohalogenation of Alkynes ${ }^{a}$

\begin{tabular}{|c|c|c|c|c|}
\hline entry & iodine(III) (equiv) & base (equiv) & solvent & yield $(\%)^{b}$ \\
\hline 1 & PIDA (1.5) & $\begin{array}{ll}---- \\
\end{array}$ & DCE & 30 \\
\hline 2 & PIDA (1.5) & ----- & DCE & $31^{c}$ \\
\hline 3 & PIDA (1.5) & ----- & DCE & $0^{d}$ \\
\hline 4 & PIDA (1.5) & ----- & DCE & $0^{e}$ \\
\hline 5 & PIDA (1.5) & $\mathrm{Cs}_{2} \mathrm{CO}_{3}(1.0)$ & DCE & 60 \\
\hline 6 & PIDA (1.5) & $\mathrm{Cs}_{2} \mathrm{CO}_{3}(1.5)$ & DCE & 67 \\
\hline 7 & PIDA (1.5) & $\mathrm{K}_{2} \mathrm{CO}_{3}(1.5)$ & DCE & 50 \\
\hline 8 & PIDA (1.5) & $\mathrm{K}_{3} \mathrm{PO}_{4}(1.5)$ & DCE & 36 \\
\hline 9 & PIDA (1.5) & $\mathrm{NaHCO}_{3}(1.5)$ & DCE & 52 \\
\hline 10 & PIDA (1.5) & $\mathrm{Na}_{2} \mathrm{CO}_{3}(1.5)$ & DCE & 46 \\
\hline 11 & PIDA (1.5) & $\operatorname{DABCO}(1.5)$ & DCE & ND \\
\hline 12 & PIDA (1.3) & $\mathrm{Cs}_{2} \mathrm{CO}_{3}(1.5)$ & DCE & 55 \\
\hline 13 & PIDA (2.0) & $\mathrm{Cs}_{2} \mathrm{CO}_{3}(1.5)$ & DCE & 73 \\
\hline 14 & PIDA (2.5) & $\mathrm{Cs}_{2} \mathrm{CO}_{3}(1.5)$ & DCE & 84 \\
\hline 16 & PIDA (2.5) & $\mathrm{Cs}_{2} \mathrm{CO}_{3}(2.0)$ & DCE & 84 \\
\hline
\end{tabular}




$\begin{array}{lcccc}17 & \text { PIDA (1.8) } & \mathrm{Cs}_{2} \mathrm{CO}_{3}(1.5) & \text { DCE } & 71 \\ 18 & \text { PIDA (2.5) } & \mathrm{Cs}_{2} \mathrm{CO}_{3}(1.5) & \text { DCM } & 54 \\ 19 & \text { PIDA (2.5) } & \mathrm{Cs}_{2} \mathrm{CO}_{3}(1.5) & \mathrm{MeCN} & 61 \\ 20 & \text { PIDA (2.5) } & \mathrm{Cs}_{2} \mathrm{CO}_{3}(1.5) & \mathrm{THF} & \mathrm{ND} \\ 21 & \text { PIDA (2.5) } & \mathrm{Cs}_{2} \mathrm{CO}_{3}(1.5) & \mathrm{H} 2 \mathrm{O} & 21 \\ 22 & \text { PIDA (2.5) } & \mathrm{Cs}_{2} \mathrm{CO}_{3}(1.5) & \mathrm{EtOAc} & 30 \\ 23 & \text { PIDA (2.5) } & \mathrm{Cs}_{2} \mathrm{CO}_{3}(1.5) & \mathrm{DMF} & 17 \\ 24 & \mathrm{PIFA}(2.5) & \mathrm{Cs}_{2} \mathrm{CO}_{3}(1.5) & \mathrm{DCE} & \mathrm{ND} \\ 25 & \mathrm{PhIO}(2.5) & \mathrm{Cs}_{2} \mathrm{CO}_{3}(1.5) & \mathrm{DCE} & \mathrm{ND} \\ 26 & \mathrm{PhI}(\mathrm{OH})(\mathrm{OTs}) & \mathrm{Cs}_{2} \mathrm{CO}_{3}(1.5) & \mathrm{DCE} & \mathrm{ND} \\ & (2.5) & & & \end{array}$

${ }^{a}$ Reaction conditions: Alkyne 1a $(1 \mathrm{mmol})$, Amine 2a (1 mmol), PIDA 3a $(2.5 \mathrm{mmol})$, Base, dry solvent $3 \mathrm{~mL}$, rt $\left(27{ }^{\circ} \mathrm{C}\right)$ for $8-18 \mathrm{~h} ;{ }^{b}$ Isolated yield after silica column chromatography; ${ }^{c} \mathrm{Na}_{2} \mathrm{SO}_{4}$ (4 equiv); ${ }^{d} \mathrm{TBAI}\left(2\right.$ equiv); ${ }^{e} \mathrm{NBS}$ (2 equiv); ND = Not detected.

\section{Results and discussion}

We choose diethyl acetylenedicarboxylate 1a, aniline $\mathbf{2 a}$ and phenyliodine(III) diacetate (PIDA) 3a as the model substrates to initiates our studies. At the beginning, the reaction was performed at room temperature in dichloromethane (DCE). To our delight, we observed the formation of desired product 4aaa in $31 \%$ yield with $(Z)$-configuration (Table 1, entry 1 ). The structure and stereochemistry of 4aaa was determined by the X-ray crystallography analysis (Supporting information, SI). With this promising result in hand, we further optimized the reaction conditions. When reaction was carried out in the presence of 4 equiv of $\mathrm{Na}_{2} \mathrm{SO}_{4}$ as additive, the product 4 aaa was yielded in $31 \%$ after $18 \mathrm{~h}$ reaction time (Table 1, entry 2). No product was obtained when the reaction was performed with TBAI and NBS as additives (entries $3 \& 4$ ). We then turned our attention to the screening of bases to improve the reaction performance. When reaction performed with $\mathrm{Cs}_{2} \mathrm{CO}_{3}$ pleasingly, the product 4 aaa was obtained in $67 \%$ yield in $12 \mathrm{~h}$ (entries 5\&6). Other bases such as $\mathrm{K}_{2} \mathrm{CO}_{3}, \mathrm{~K}_{3} \mathrm{PO}_{4}, \mathrm{NaHCO}_{3}, \mathrm{Na}_{2} \mathrm{CO}_{3}$, and DABCO did not improve the yield of the product (entries 7-11). We then screened the equivalents of PIDA and $\mathrm{Cs}_{2} \mathrm{CO}_{3}$ (entries 12-17), 1.5 equiv of $\mathrm{Cs}_{2} \mathrm{CO}_{3}$ and 2.5 equiv 
of PIDA were found to be the best conditions to afford the product 4 aaa in $84 \%$ yield after $8 \mathrm{~h}$ (entry 14). Among the solvents tested, DCE was found to be the best solvent choice (entries 18-23). In our attempts to further improve the yield, we have screened other organoiodine sources, unfortunately our attempts went in vain (entries 24-26).

In order to realize the versatility of this newly developed method, we anticipated to apply it to a series of amines having neutral, electron donating and withdrawing substituents on phenyl ring. This results demonstrated that the substrates containing neutral and electron donating groups on phenyl ring afforded the products (Table 2(A)) in good to very good yield. The weak electron withdrawing substituents on phenyl ring resulted in relatively low yields (Table 2(B)). Presence of both weak electron withdrawing and donating groups on the same phenyl ring gave the products fairly in good yields (Table 2, $\mathbf{4 f a b} \& \mathbf{4 m a a}$ ). Organoiodine with strong electron withdrawing groups $\left(-\mathrm{CO}_{2} \mathrm{Me}, \mathrm{NO}_{2}\right)$ gave the products in excellent yields (Table 2(C)), this may be due to stabilization of intermediate $\mathbf{V}$ (Scheme 5). It is worth to mention here that, the robustness of our strategy is demonstrated by the synthesis of derivatives (Table 2 (D), 4a"aa \& 4a"ab) involving formation of four new C-N bonds and two C-I bonds in single step. After developing successful syntheses of halogenated enamines, we envisaged that it would be appropriate to check the scalability of our protocol for the synthesis halogenated enamines owing to their synthetic utility. Accordingly, we performed the reaction on gram scale for the synthesis of halogenated enamine resulted in $72 \%$ yield (Table 2 , 4aaa).

Table 2. Substrate Scope of Aminoiodination Reaction ${ }^{a}$

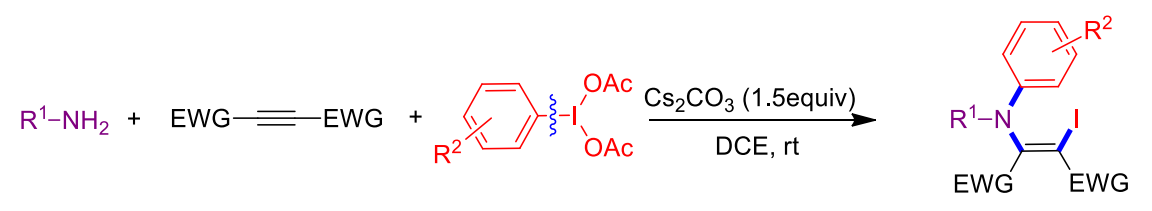


(A) Scope of Arylamines with neutral and electron donating substituents and Alkynes

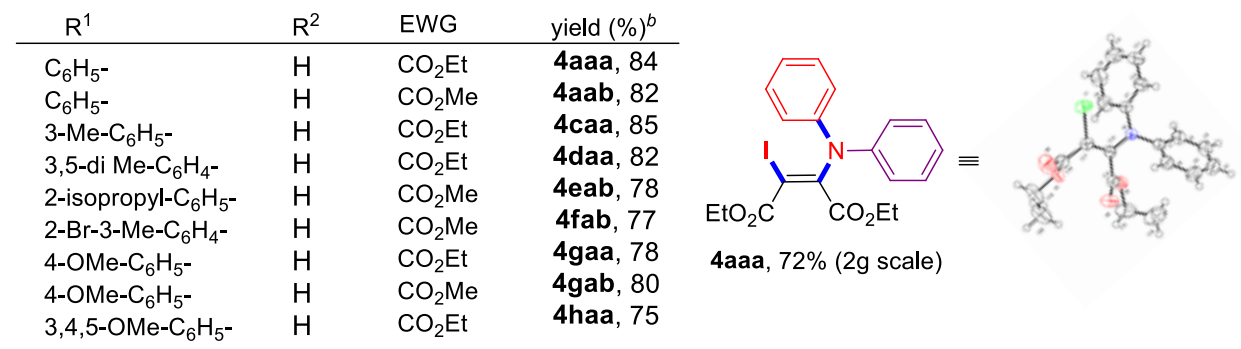

(B) Scope of Arylamines with weak electron withdrawing goups and Alkynes

\begin{tabular}{llll}
\multicolumn{1}{c}{$\mathrm{R}^{1}$} & $\mathrm{R}^{2}$ & $\mathrm{EWG}$ & yield (\%) \\
\hline $2-\mathrm{F}-\mathrm{C}_{6} \mathrm{H}_{5^{-}}$ & $\mathrm{H}$ & $\mathrm{CO}_{2} \mathrm{Me}$ & 4iab, 88 \\
$4-\mathrm{Cl}-\mathrm{C}_{6} \mathrm{H}_{5^{-}}$ & $\mathrm{H}$ & $\mathrm{CO}_{2} \mathrm{Et}$ & 4jaa, 85 \\
$4-\mathrm{Br}-\mathrm{C}_{6} \mathrm{H}_{5^{-}}$ & $\mathrm{H}$ & $\mathrm{CO}_{2} \mathrm{Et}$ & $\mathbf{4 k a a}, 88$ \\
$4-\mathrm{Br}-\mathrm{C}_{6} \mathrm{H}_{5^{-}}$ & $\mathrm{H}$ & $\mathrm{CO}_{2} \mathrm{Me}$ & $\mathbf{4 k a b}, 83$ \\
$2-\mathrm{Br}-\mathrm{C}_{6} \mathrm{H}_{5-}^{-}$ & $\mathrm{H}$ & $\mathrm{CO}_{2} \mathrm{Et}$ & $\mathbf{4 l a a}, 79$ \\
$2-\mathrm{Me}, 3-\mathrm{NO}_{2}-\mathrm{C}_{6} \mathrm{H}_{5^{-}}$ & $\mathrm{H}$ & $\mathrm{CO}_{2} \mathrm{Et}$ & 4 maa, 75
\end{tabular}

(C) Scope of Organoiodine(III) reagents and Alkynes

\begin{tabular}{|c|c|c|c|}
\hline $\mathrm{R}^{1}$ & $R^{2}$ & EWG & yield $(\%)^{b}$ \\
\hline $\mathrm{C}_{6} \mathrm{H}_{5^{-}}$ & $\mathrm{CO}_{2} \mathrm{Me}$ & $\mathrm{CO}_{2} \mathrm{Et}$ & 4aba, 90 \\
\hline $\mathrm{C}_{6} \mathrm{H}_{5^{-}}$ & $\mathrm{CO}_{2} \mathrm{Me}$ & $\mathrm{CO}_{2} \mathrm{Me}$ & $4 a b b, 87$ \\
\hline 4-OMe- $\mathrm{C}_{6} \mathrm{H}_{5}-$ & $\mathrm{CO}_{2} \mathrm{Me}$ & $\mathrm{CO}_{2} \mathrm{Me}$ & 4gbb, 77 \\
\hline $4-\mathrm{Br}-\mathrm{C}_{6} \mathrm{H}_{5^{-}}$ & $\mathrm{CO}_{2} \mathrm{Me}$ & $\mathrm{CO}_{2} \mathrm{Me}$ & 4kbb, 91 \\
\hline 2-Br-4-Me- $\mathrm{C}_{6} \mathrm{H}_{4}-$ & $\mathrm{CO}_{2} \mathrm{Me}$ & $\mathrm{CO}_{2} \mathrm{Et}$ & $4 n b a, 86$ \\
\hline $\mathrm{C}_{6} \mathrm{H}_{5^{-}}$ & $3-\mathrm{NO}_{2}$ & $\mathrm{CO}_{2} \mathrm{Et}$ & 4aca, 84 \\
\hline $\mathrm{C}_{6} \mathrm{H}_{5}-$ & $4-\mathrm{F}$ & $\mathrm{CO}_{2} \mathrm{Me}$ & $4 \mathrm{adb}, 90$ \\
\hline 2,4-di Me- $\mathrm{C}_{6} \mathrm{H}_{4-}$ & $4-\mathrm{F}$ & $\mathrm{CO}_{2} \mathrm{Et}$ & 4oda, 87 \\
\hline
\end{tabular}

(D) Scope of aliphatic amines and Alkynes
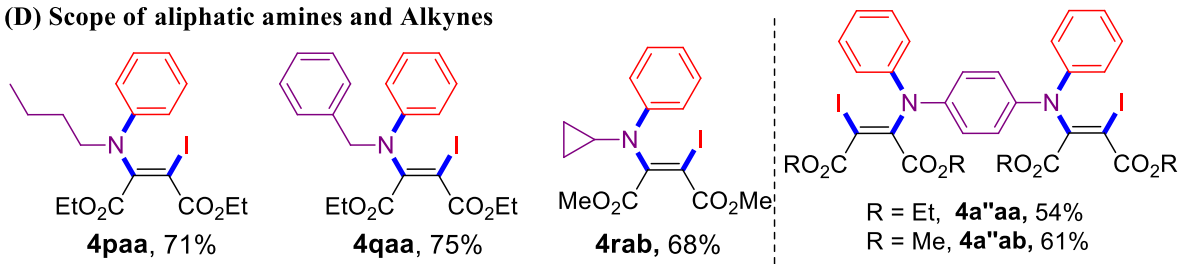

${ }^{a}$ Reaction conditions: Alkyne 1a $(1 \mathrm{mmol})$, Amine 2a $(1 \mathrm{mmol})$, PIDA 3a $(2.5 \mathrm{mmol}), \mathrm{Cs}_{2} \mathrm{CO}_{3}(1.5 \mathrm{mmol})$, dry $\mathrm{DCE}$ $3 \mathrm{~mL}$, rt $\left(27^{\circ} \mathrm{C}\right)$ for $8-14 \mathrm{~h} ;{ }^{b}$ Isolated yield after silica column chromatography.

Owing to the importance of halogenated enamines as flexible building blocks in organic synthesis, we envisioned to employ them in organic transformations. In this direction, we thought to perform transition-metal catalyzed intramolecular cyclizations by $\mathrm{C}-\mathrm{I}$ and $\left(\mathrm{sp}^{2}\right) \mathrm{C}-\mathrm{H}$ activation. However, less reactive $3^{\circ}$ enamines than $2^{\circ}$ enamine homologues in transition-metal catalyzed $\mathrm{C}-\mathrm{H}$ activation reactions are scarce ${ }^{17}$ and poses a daunting challenge. The conversion of such enamines to indoles was accomplished by improving the electrophilicity of Pd catalysts under acidic conditions. ${ }^{18}$ 
Herein we delighted to employ these iodinated enamines for smooth transformation to $N$-aryl indoles via palladium-catalyzed intramolecular cyclization under non-acidic conditions. It was noted that iodinated enamines containing weak electron withdrawing and electron donating groups on phenyl rings provided the indoles with good yields than those having electron-withdrawing groups (Scheme 3).

\section{Scheme 3. $N$-aryl Indole Synthesis from Iodinated Enamines via Pd-Catalyzed Intramolecular Cyclizaiton $^{a}$}

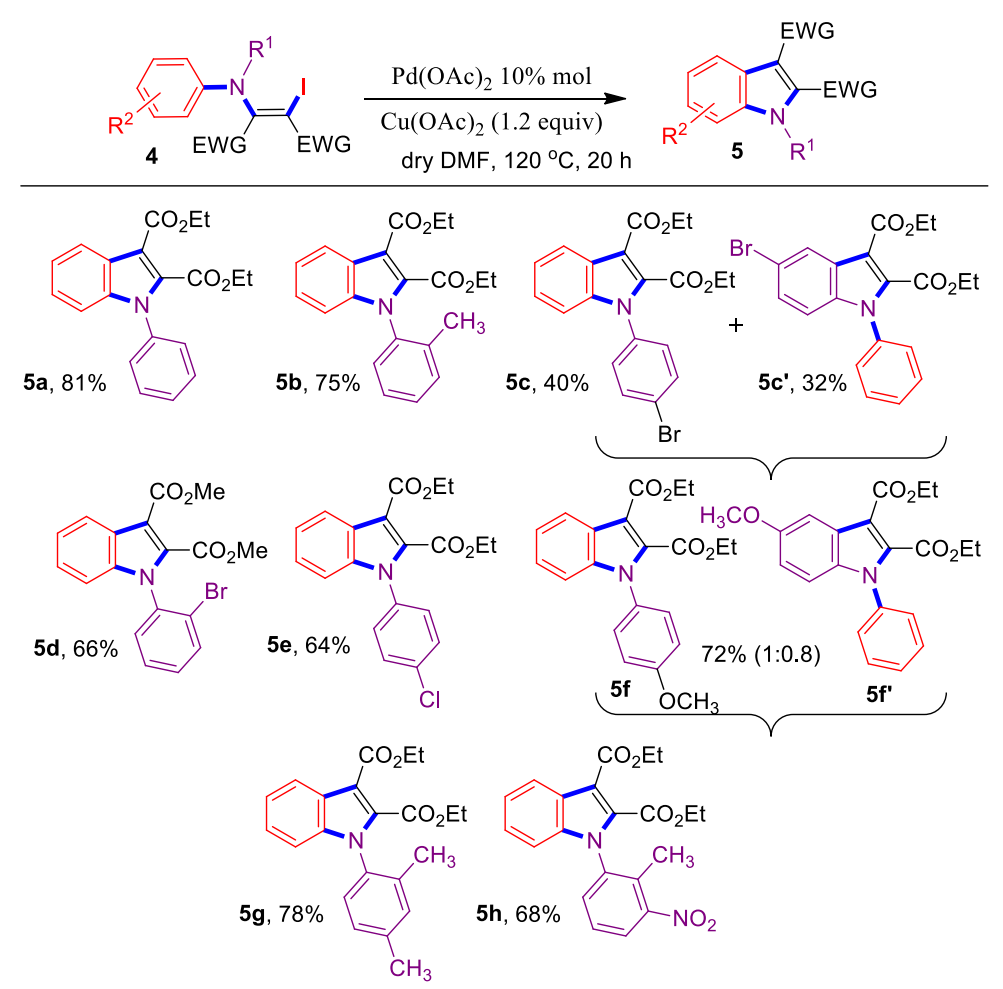

$\overline{{ }^{a} \text { Reaction conditions: Iodoenamine } 4(1 \mathrm{mmol}), \mathrm{Pd}(\mathrm{OAc})_{2}(10 \% \mathrm{~mol}), \mathrm{Cu}(\mathrm{OAc})_{2}(1.2 \mathrm{mmol}), \mathrm{Cs}_{2} \mathrm{CO}_{3}(1.5 \mathrm{mmol}), \text { dry DMF } 1.5}$ $\mathrm{mL}$, at $120^{\circ} \mathrm{C}$ for 20 h. ${ }^{b}$ Isolated yield after silica column chromatography. ${ }^{c}$ Ratio estimated by $\mathrm{H}^{1} \mathrm{NMR}$.

To investigate the reaction mechanism, we performed couple of control experiments (Scheme 4). The addition of TEMPO uninhibited the formation of 4aaa (Scheme 4(i)), suggesting that reaction might proceed through ionic pathway. To further rule out the radical pathway, we performed a radical clock experiment under standard conditions, which afforded only the halogenated enamine product 4rab and no other ring-opened coupling products (Scheme 4(ii)). 


\section{Scheme 4. Control Experiments}

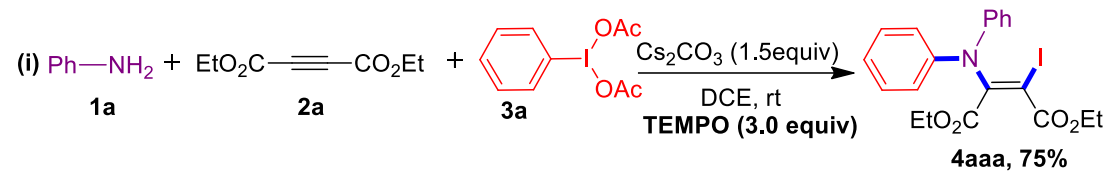

(ii) Radical clock experiment:

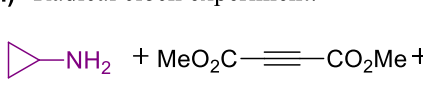

$1 \mathrm{u}$

2b
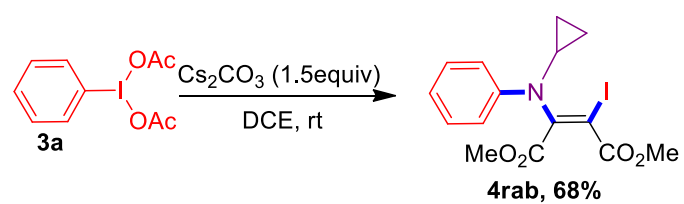

Based on the above experiments and literature reports ${ }^{9}$ we have formulated the following plausible mechanism (scheme 5). The intermolecular reaction of 1a and 2a generates hydroamination product I which undergo ligand exchange with PIDA by the loss of acetic acid to give intermediate II, which would further transformed to intermediate (IV) by the loss of one more acetic acid, which followed by subsequent rearrangement leads to the formation of a zwitterion $(\mathbf{V})$ which then afford the final product 4aaa.

\section{Scheme 5. Plausible Mechanism for Aminohalogenation of Alkynes}
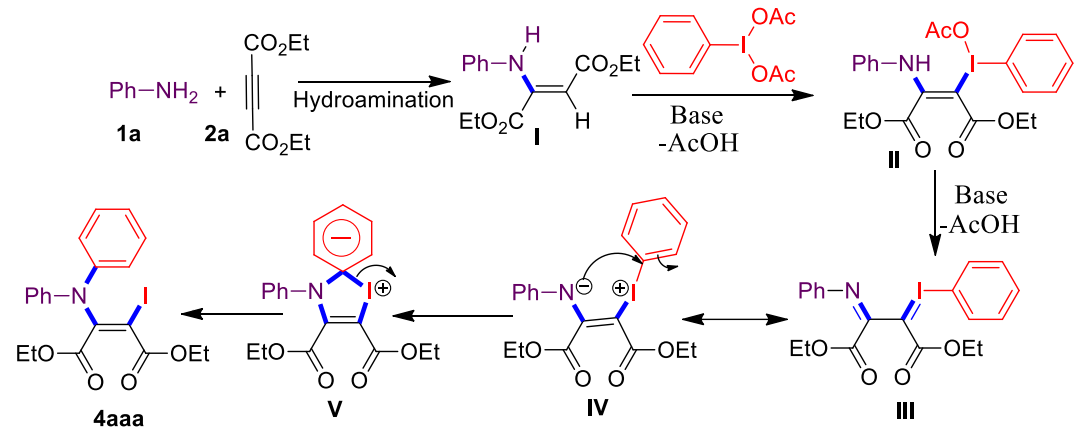

In summary, we have developed a novel and facile approach for the stereoselective alkyne aminoiodination using amines, phenyliodine(III) diacetate (PIDA) as iodide and aryl moiety source as well as promoter. The key feature of this reaction is the incorporation of iodide and aryl group concurrently in the same molecule in a stereoselective manner. The advantages of this method are metal-free, mild reaction conditions and scalability. We have also successfully employed these inactive iodinated $3^{\circ}$ enamines in intramolecular cyclizations via Pd catalyzed C-H and C-I activation resulting in highly functionalized indoles. Further exploration of iodinated $3^{\circ}$ enamines are currently under way in our laboratory. 


\section{Supporting Information}

The Supporting Information is available free of charge on the ACS Publications website.

Experimental procedures, characterization data, and copies of NMR (PDF).

\section{AUTHOR INFORMATION}

\section{Corresponding Author}

*E-mail: sharada@iith.ac.in

\section{ACKNOWLEDGMENT}

We gratefully acknowledge the Department of Science and Technology-Science and Engineering Research Board (DST-SERB-EMR/2016/1000952) New Delhi, India and Indian Institute of Technology Hyderabad (IITH) for financial support. S.A thanks CSIR, New Delhi, India for the award of research fellowship.

\section{Experimental Section}

General Considerations: IR spectra were recorded on a FTIR spectrophotometer. ${ }^{1} \mathrm{H}$ NMR spectra were recorded on $400 \mathrm{MHz}$ spectrometer at $295 \mathrm{~K}$ in $\mathrm{CDCl}_{3}$; chemical shifts ( $\left.\delta \mathrm{ppm}\right)$ and coupling constants $(\mathrm{Hz})$ are reported in standard fashion with reference to either internal standard tetramethylsilane (TMS) $\left(\delta_{\mathrm{H}}\right.$ $=0.00 \mathrm{ppm})$ or $\mathrm{CHCl}_{3}\left(\delta_{\mathrm{H}}=7.25 \mathrm{ppm}\right) .{ }^{13} \mathrm{C} \mathrm{NMR}$ spectra were recorded on $100 \mathrm{MHz}$ spectrometer at 25 ${ }^{\circ} \mathrm{C}$ in $\mathrm{CDCl}_{3}$; chemical shifts $(\delta \mathrm{ppm})$ are reported relative to $\mathrm{CHCl}_{3}\left[\delta_{\mathrm{C}}=77.00 \mathrm{ppm}\right.$ (central line of triplet)]. In the ${ }^{1} \mathrm{HNMR}$, the following abbreviations were used throughout: $\mathrm{s}=$ singlet, $\mathrm{d}=$ doublet, $\mathrm{t}=$ triplet, $\mathrm{q}=$ quartet, qui $=$ quintet, $\mathrm{dd}=$ doublet of doublets, $\mathrm{m}=$ multiplet and $\mathrm{br} \mathrm{s} .=$ broad singlet. The assignment of signals was confirmed by ${ }^{1} \mathrm{H},{ }^{13} \mathrm{C} \mathrm{CPD}$, and DEPT spectra. High-resolution mass spectra (HR-MS) were recorded using Q-TOF multimode source. Melting points were determined on an electrothermal melting point apparatus and are uncorrected. Hypervalent iodine reagents phenyliodine bis(trifluoroacetate) (PIFA) and phenyliodine diacetate (PIDA) were purchased from Sigma Aldrich. Other reagents were purchased as reagent grade and used without further purification. All dry solvents were used. $\mathrm{CH}_{3} \mathrm{CN}$ and DCE were dried over $\mathrm{CaH}_{2}$, DCM \& DMF were dried over anhydrous $\mathrm{P}_{2} \mathrm{O}_{5}$, and ethyl acetate (EtOAc) was dried over $\mathrm{Na}_{2} \mathrm{CO}_{3}$ and distilled before use. 
All small scale dry reactions were carried out using standard syringe-septum technique. Reactions were monitored by TLC on silica gel using a combination of petroleum ether and ethyl acetate as eluents. All reactions were performed under air atmosphere in standard glassware, heated at $80{ }^{\circ} \mathrm{C}$ for $3 \mathrm{~h}$ before use. Solvents were distilled prior to use; petroleum ether with a boiling range of 40 to $60{ }^{\circ} \mathrm{C}$ was used. Organic solutions were concentrated by rotary evaporation under vacuum. Acme's silica gel (60-120 mesh) was used for column chromatography (approximately $20 \mathrm{~g}$ per one gram of crude material).

\section{General Procedure-I for Aminoiodination of Alkynes Using Synthesis of 4aaa as an Example.}

Aniline $1 \mathbf{a}(50 \mathrm{mg}, 1 \mathrm{mmol})$ was taken in a dried round bottom flask, and dialkyl acetylenedicarboxylate 2a (91.3 mg, $1 \mathrm{mmol}$ ) was then added slowly with thorough mixing to form a homogeneous paste, then add $0.5 \mathrm{~mL}$ DCE solvent (if required). After confirming the formation of enamine (monitored by TLC), then added $4 \mathrm{~mL}$ DCE solvent, followed by addition of $\mathrm{Cs}_{2} \mathrm{CO}_{3}(261.959 \mathrm{mg}, 1.5 \mathrm{mmol})$, phenyliodine(III) diacetate (PIDA) 3a (431.614 mg, $2.5 \mathrm{mmol}$ ) in portion wise for $20 \mathrm{~min}$. The progress of the reaction was monitored by TLC. The reaction mixture was quenched by addition of saturated solution of $\mathrm{NaHCO}_{3}$ and extracted with ethyl acetate (EtOAc), dried over $\mathrm{MgSO}_{4}$, and concentrated in vacuo. The residue was purified through a silica gel column chromatography using petroleum ether/ethyl acetate (0.2/9.8) as eluent to yield (209 mg, 84\%). All compounds (4aaa-4rab) were unknown and confirmed by FTIR, ${ }^{1} \mathrm{H}$ NMR, ${ }^{13} \mathrm{C}$ NMR and HR-MS spectral analyses.

\section{General Procedure-II for Aminoiodination of Alkyne Using Synthesis of 4a"aa as an Example.}

p-Phenylenediamine 1a" $(50 \mathrm{mg}, 1 \mathrm{mmol})$ was taken in a dried round bottom flask, and dialkyl acetylenedicarboxylate $\mathbf{2 a}(157.35 \mathrm{mg}, 2 \mathrm{mmol})$ was then added slowly with thorough mixing to form a homogeneous paste, then add $2 \mathrm{~mL}$ DCE solvent (if required). After confirming the formation of enamine (monitored by TLC), then added $8 \mathrm{~mL}$ DCE solvent, followed by addition of $\mathrm{Cs}_{2} \mathrm{CO}_{3}(451.58 \mathrm{mg}, 3.0$ mmol), phenyliodine(III) diacetate (PIDA) 3a $(744.05 \mathrm{mg}, 5.0 \mathrm{mmol})$ in portion wise for $20 \mathrm{~min}$. The progress of the reaction was monitored by TLC. The reaction mixture was quenched by addition of saturated solution of $\mathrm{NaHCO}_{3}$ and extracted with ethyl acetate (EtOAc), dried over $\mathrm{MgSO}_{4}$, and concentrated in 
vacuo. The residue was purified through a silica gel column chromatography using petroleum ether/ethyl acetate (0.2/9.8) as eluent to yield (212.55 mg, 54\%). Compounds 4a"aa and 4a"ab were unknown and confirmed by FTIR, ${ }^{1} \mathrm{H}$ NMR, ${ }^{13} \mathrm{C}$ NMR and HR-MS spectral analyses.

\section{General Procedure-III for the Synthesis of N-Aryl Indoles Using Synthesis of 5a as an Example.}

To a mixture of $N$-diaryl $\beta$-iodinated enamine $4 \mathbf{a}(50 \mathrm{mg}, 1.0 \mathrm{mmol}), \mathrm{Pd}(\mathrm{OAc})_{2}(2.4 \mathrm{mg}, 0.1 \mathrm{mmol})$ and $\mathrm{Cu}(\mathrm{OAc})_{2}(23.32 \mathrm{mg}, 1.2 \mathrm{mmol})$ was added $1.5 \mathrm{~mL}$ of dry DMF in an oven dried $15 \mathrm{~mL}$ schlenk tube. The reaction mixture was stirred at $120^{\circ} \mathrm{C}$ for $20 \mathrm{~h}$. After cooling the reaction mixture to room temperature, then the reaction mixture was quenched by addition of water and extracted with ethyl acetate followed by water wash for two times, dried over $\mathrm{MgSO}_{4}$, and concentrated in vacuo. The residue was purified by silica gel column chromatography using petroleum ether/ethyl acetate $(0.2 / 9.8)$ as eluent to yield $N$-Aryl indole product 5a (26.82 mg, 74\%). Compounds 5a-5h were unknown and confirmed by FTIR, ${ }^{1} \mathrm{H}$ NMR, ${ }^{13} \mathrm{C}$ NMR and HR-MS spectral analyses.

\section{General procedure (GP-IV) for the synthesis organoiodine(III) reagents $(\mathbf{3 b}, \mathbf{3 c} \& \mathbf{3 d})^{19}$ :}

Compounds $\mathbf{3 b}, \mathbf{3 c}$ and $\mathbf{3 d}$ were prepared according to the literature method. ${ }^{18}$ To a solution of substituted iodobenzene $(1.0 \mathrm{mmol})$ in $\mathrm{AcOH}$ was added $m \mathrm{CPBA}(\mathrm{ca} .65 \%, 1.15 \mathrm{mmol})$. The mixture was stirred at room temperature for $2 \mathrm{~h}$. Then, $\mathrm{H}_{2} \mathrm{O}$ was added to the reaction mixture and then it was extracted with $\mathrm{CHCl}_{3}$. After being dried over $\mathrm{Na}_{2} \mathrm{SO}_{4}$, filtration, and removal of the solvent $\mathrm{Et}_{2} \mathrm{O}$ and hexane were added to the residue, and the mixture was cooled to $0^{\circ} \mathrm{C}$ to induce precipitation. After filtration, the solids were washed with a mixture of $\mathrm{Et}_{2} \mathrm{O}$ and hexane to provide products (Diacetoxyiodo)benzene derivatives (3b, 3c and 3d).

\section{(Z)Diethyl 2-(diphenylamino)-3-iodomaleate (4aaa)}

Deep yellow solid $(209 \mathrm{mg}, 84 \%)$. m.p. $=54-56{ }^{\circ} \mathrm{C}$ (recrystallized by placing inside the refrigerator); FRIR (MIR-ATR, 4000-600 cm-1) $v_{\max }=3034.9,2980.5,2936.4,1729.9,1491.7,1288.1,1219.4,1041.4$, 1027.6, 838.2, 756.9, 695.1; ${ }^{1} \mathrm{H}$ NMR $\left(\mathrm{CDCl}_{3}, 400 \mathrm{MHz}\right): \delta_{\mathrm{H}}=7.30(\mathrm{~m}, 4 \mathrm{H}) ; 7.11(\mathrm{~m}, 6 \mathrm{H}) ; 4.26(\mathrm{q}, J=7.2$ 
$\mathrm{Hz}, 2 \mathrm{H}) ; 4.02(\mathrm{q}, J=6.8 \mathrm{~Hz}, 2 \mathrm{H}) ; 1.33(\mathrm{t}, J=7.1 \mathrm{~Hz}, 3 \mathrm{H}) ; 0.96(\mathrm{t}, J=7.1 \mathrm{~Hz}, 3 \mathrm{H}) ;{ }^{13} \mathrm{C} \mathrm{NMR}\left(\mathrm{CDCl}_{3}, 100\right.$ MHz): 164.3, 145.4, 144.5, 142.8, 141.8, 141.3, 133.3, 1130.5, 102.6, 63.1, 14 .1; HR-MS (ESI+) m/z value calculated for $\left[\mathrm{C}_{20} \mathrm{H}_{20} \mathrm{INO}_{4}\right]^{+}=[\mathrm{M}+\mathrm{H}]^{+}: 466.0510$; found: 466.0498 .

\section{(Z)Dimethyl 2-(diphenylamino)-3-iodomaleate (4aab)}

Yellow oil (197 mg, 82\%); FT-IR: (MIR-ATR, 4000-600 $\left.\mathrm{cm}^{-1}\right): \mathrm{v}_{\max }=3033.1,3006.6,2949.9,2849.1$, 1732.8, 1550.3, 1489.7, 1434.1, 1288.7, 1223.3, 1147.4, 1042.8, 988.8, 757.4, 695.2; ${ }^{1} \mathrm{H}$ NMR $\left(\mathrm{CDCl}_{3}, 400\right.$ $\mathrm{MHz}): \delta_{\mathrm{H}}=7.30(\mathrm{~m}, 4 \mathrm{H}) ; 7.1(\mathrm{~m}, 6 \mathrm{H}) ; 3.80(\mathrm{~s}, 3 \mathrm{H}) ; 3.57(\mathrm{~s}, 3 \mathrm{H}) ;{ }^{13} \mathrm{C} \mathrm{NMR}\left(\mathrm{CDCl}_{3}, 100 \mathrm{MHz}\right): 166.2$, 164.5, 149.5, 144.1, 129.3, 124.7, 124.0, 86.9, 53.5, 52.9; HR-MS (ESI+) m/z value calculated for $\left[\mathrm{C}_{18} \mathrm{H}_{16} \mathrm{IKNO}_{4}\right]^{+}=[\mathrm{M}+\mathrm{K}]^{+}:$475.9756; found: 475.9749 .

\section{(Z)Diethyl 2-iodo-3-(phenyl(m-tolyl)amino)maleate (4caa)}

Yellow oil (190 mg, 85\%); FT-IR: (MIR-ATR, 4000-600 $\left.\mathrm{cm}^{-1}\right): \mathrm{V}_{\max }=2936.66,1731.04,1701.04,1592.15$, 1503.53, 1459.87, 1421.39, 1234.69, 1206.48, 1128.33, 1008.78, 759.89, 699.87; ${ }^{1} \mathrm{H} \mathrm{NMR}\left(\mathrm{CDCl}_{3}, 400\right.$ MHz): $\delta_{\mathrm{H}}=7.26-7.32(\mathrm{~m}, 2 \mathrm{H}), 7.14-7.19(\mathrm{~m}, 1 \mathrm{H}), 7.10(\mathrm{~d}, J=6.85 \mathrm{~Hz}, 3 \mathrm{H}), 6.87-6.93(\mathrm{~m}, 3 \mathrm{H}), 4.24$ $(\mathrm{q}, J=7.17 \mathrm{~Hz}, 2 \mathrm{H}), 4.02(\mathrm{q}, J=7.34 \mathrm{~Hz}, 2 \mathrm{H}), 2.29(\mathrm{~s}, 3 \mathrm{H}), 1.31(\mathrm{t}, J=7.09 \mathrm{~Hz}, 3 \mathrm{H}), 0.97$ (t, $J=7.09 \mathrm{~Hz}$, $3 \mathrm{H}) ;{ }^{13} \mathrm{C} \mathrm{NMR}\left(\mathrm{CDCl}_{3}, 100 \mathrm{MHz}\right): 165.9,163.9,149.5,144.3,144.2,139.1,129.2,129.0,125.4,124.6$, $124.4,124.2,121.2,87.4,62.6,62.0,21.4,14.0,13.6$; HR-MS (ESI+) $\mathrm{m} / \mathrm{z}$ value calculated for $\left[\mathrm{C}_{21} \mathrm{H}_{23} \mathrm{INO}_{4}\right]^{+}=[\mathrm{M}+\mathrm{H}]^{+}:$480.0670; found: 480.0672 .

\section{(Z)Diethyl 2-((3,5-dimethylphenyl)(phenyl)amino)-3-iodomaleate (4daa)}

Yellow oil (166 mg, 82\%); FT-IR: (MIR-ATR, 4000-600 $\mathrm{cm}^{-1}$ ): $\mathrm{V}_{\max }=2998.4,2945.1,2818.4,1718.9$,

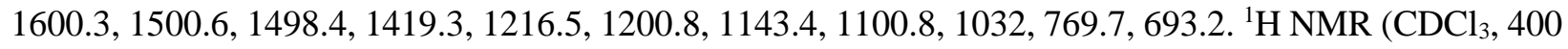
MHz): $\delta_{\mathrm{H}}=7.23(\mathrm{~d}, J=6.4 \mathrm{~Hz}, 2 \mathrm{H}) ; 7.07,(\mathrm{~m}, 2 \mathrm{H}) ; 6.97(\mathrm{~m}, 3 \mathrm{H}) ; 6.83(\mathrm{br}, \mathrm{s}, 1 \mathrm{H}) ; 4.21(\mathrm{~d}, J=7.3 \mathrm{~Hz}, 2 \mathrm{H})$; $3.98(\mathrm{~d}, J=7.3 \mathrm{~Hz}, 2 \mathrm{H}) ; 2.28(\mathrm{~s}, 3 \mathrm{H}) ; 2.04(\mathrm{~s}, 3 \mathrm{H}) ; 1.31(\mathrm{t}, J=7.04 \mathrm{~Hz}, 3 \mathrm{H}) ; 0.96(\mathrm{t}, J=7.04 \mathrm{~Hz}, 3 \mathrm{H}) .{ }^{13} \mathrm{C}$ $\mathrm{NMR}\left(\mathrm{CDCl}_{3}, 100 \mathrm{MHz}\right): \delta=165.8,164.2,151.7,144.8,141.2,136.2,133.7,131.4,129.0,128.8,127.7$, 123.9, 123.2, 80.2, 62.5, 61.9, 21.7, 20.9, 18.8, 14, 13.5; HR-MS (ESI+) m/z value calculated for $\left[\mathrm{C}_{22} \mathrm{H}_{25} \mathrm{INO}_{4}\right]^{+}=[\mathrm{M}+\mathrm{H}]^{+}:$494.0828; found: 494.0824 .

\section{(Z)Dimethyl 2-iodo-3-((2-isopropylphenyl)(phenyl)amino)maleate (4eab)}

Yellow oil (138 mg, 78\%); FT-IR: (MIR-ATR, 4000-600 $\mathrm{cm}^{-1}$ ): $\mathrm{V}_{\max }=2997.4,2918.4,1712.9,1683.3$, 1501.6, 1496.4, 1427.3, 1215.4, 1202.6, 1133.4, 1022.4, 767.7, 694.2; ${ }^{1} \mathrm{H} \mathrm{NMR}\left(\mathrm{CDCl}_{3}, 400 \mathrm{MHz}\right): \delta_{\mathrm{H}}=$ 7.31 - 7.35 (m, 1H), 7.23 - 7.30 (m, 3H), 7.11 - $7.18(\mathrm{~m}, 2 \mathrm{H}), 7.06$ (t, J = 7.34 Hz, 1H), 6.94 - 7.04 (m, 1H), 6.86 (br. s., 1H), 3.76 (s, 3H), 3.50 (s, 3H), 3.17 (sept, $J=6.85 \mathrm{~Hz}, 1 \mathrm{H}), 1.04-1.24(\mathrm{~d}, 6 \mathrm{H}),{ }^{13} \mathrm{C}$ NMR 
$\left(\mathrm{CDCl}_{3}, 100 \mathrm{MHz}\right): \delta=166.0,164.8,152.9,147.2,145.5,140.6,129.3,129.0,128.0,127.4,126.6,124.4$, 124.2, 53.5, 52.7, 28.0; HR-MS (ESI+) $\mathrm{m} / \mathrm{z}$ value calculated for $\left[\mathrm{C}_{22} \mathrm{H}_{24} \mathrm{INO}_{4}\right]^{+}=[\mathrm{M}+\mathrm{H}]^{+}: 480.0672$; found: 480.0668 .

\section{(Z)Dimethyl 2-((2-bromo-5-methylphenyl)(phenyl)amino)-3-iodomaleate (4fab)}

Yellow oil (110 mg, 77\%); FT-IT:: (MIR-ATR,4000-600 cm-1): $\mathrm{v}_{\max }=3002.8,2928.4,2890.4,1720.6$, $1555.7,1429.7,1420,1230.6,1078.9,985.7,760.5,695.3 ;{ }^{1} \mathrm{H} \mathrm{NMR}\left(\mathrm{CDCl}_{3}, 400 \mathrm{MHz}\right): \delta_{\mathrm{H}}=7.42(\mathrm{~s}, 1 \mathrm{H})$; $7.22(\mathrm{~d}, J=7.8 \mathrm{~Hz}, 2 \mathrm{H}) ; 7.12(\mathrm{~d}, J=8.3 \mathrm{~Hz}, 3 \mathrm{H}) ; 7.08(\mathrm{~s}, 1 \mathrm{H}) ; 7.06(\mathrm{~s}, 1 \mathrm{H}) ; 3.78(\mathrm{~s}, 3 \mathrm{H}) ; 3.55$ (s, $3 \mathrm{H}) ; 2.34$ (s, 3H); ${ }^{13} \mathrm{C} \mathrm{NMR}\left(\mathrm{CDCl}_{3}, 100 \mathrm{MHz}\right): \delta=166.1,164.5,151.2,144.3,139.3,138.3,134.7,129.7,129.4$, 129.1, 124.0, 123.1, 82.2, 53.5, 52.8, 20.8; HR-MS (ESI+) $\mathrm{m} / \mathrm{z}$ value calculated for $\left[\mathrm{C}_{19} \mathrm{H}_{17} \mathrm{BrINaNO}_{4}\right]^{+}=$ $[\mathrm{M}+\mathrm{Na}]^{+}:$553.9260; found: 553.9251 .

\section{(Z)Diethyl 2-iodo-3-((4-methoxyphenyl)(phenyl)amino)maleate (4gaa)}

Yellow oil (157 mg, 78\%); FT-IR: (MIR-ATR, 4000-600 $\left.\mathrm{cm}^{-1}\right): \mathrm{V}_{\max }=2981.42,1729.22,1555.32,1490.18$, 1290.53, 1227.06, 1193.16, 1043.82, 758.67, 694.93; ${ }^{1} \mathrm{H} \mathrm{NMR}\left(\mathrm{CDCl}_{3}, 400 \mathrm{MHz}\right): \delta_{\mathrm{H}}=7.24-7.30(\mathrm{~m}$, 2H), $6.99-7.10(\mathrm{~m}, 5 \mathrm{H}), 6.81-6.87(\mathrm{~m}, 2 \mathrm{H}), 4.23(\mathrm{q}, J=7.01 \mathrm{~Hz}, 2 \mathrm{H}), 4.02(\mathrm{q}, J=7.34 \mathrm{~Hz}, 2 \mathrm{H}), 3.80(\mathrm{~s}$, $3 \mathrm{H}), 1.31(\mathrm{t}, J=7.09 \mathrm{~Hz}, 3 \mathrm{H}), 0.99(\mathrm{t}, J=7.09 \mathrm{~Hz}, 3 \mathrm{H}) ;{ }^{13} \mathrm{C} \mathrm{NMR}\left(\mathrm{CDCl}_{3}, 100 \mathrm{MHz}\right): 165.8,164.1,157.1$, 150.2 , 144.8, 137.1, 129.2, 126.7, 124.0, 123.3, 114.4, 85.0, 62.6, 62.0, 55.5, 14.0, 13.6; HR-MS (ESI+) $\mathrm{m} / \mathrm{z}$ value calculated for $\left[\mathrm{C}_{21} \mathrm{H}_{22} \mathrm{IKNO}_{5}\right]^{+}=[\mathrm{M}+\mathrm{K}]^{+}: 534.0174$; found: 534.0192

\section{(Z)Dimethyl 2-iodo-3-((4-methoxyphenyl)(phenyl)amino)maleate (4gab)}

Yellow oil (152 mg, 80\%); FT-IR: (MIR-ATR, 4000-600 $\left.\mathrm{cm}^{-1}\right): \mathrm{V}_{\max }=2950.15,2837.74,1731.23,1546.86$, 1505.44, 1433.68, 1242.06, 1220.03, 1145.92, 1031.65, 835.86, 758.74, 693.82; ${ }^{1} \mathrm{H} \mathrm{NMR}\left(\mathrm{CDCl}_{3}, 400\right.$ MHz): $\delta_{\mathrm{H}}=7.25-7.28(\mathrm{~m}, 3 \mathrm{H}), 7.07(\mathrm{~d}, J=8.80 \mathrm{~Hz}, 2 \mathrm{H}), 7.01(\mathrm{~d}, J=7.82 \mathrm{~Hz}, 2 \mathrm{H}), 6.84(\mathrm{~d}, J=8.80 \mathrm{~Hz}$,

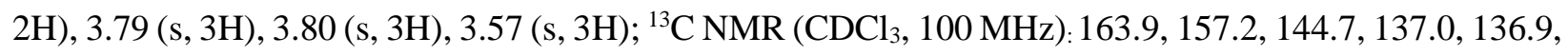
129.2, 126.7, 124.2, 123.4, 114.5, 55.5, 53.5, 52.8; HR-MS (ESI+) $\mathrm{m} / \mathrm{z}$ value calculated for $\left[\mathrm{C}_{19} \mathrm{H}_{18} \mathrm{IKNO}_{5}\right]^{+}$ $=[\mathrm{M}+\mathrm{K}]^{+}:$505.9860; found: 505.9868.

\section{(Z)Diethyl 2-iodo-3-(phenyl(3,4,5-trimethoxyphenyl)amino)maleate (4haa)}

Yellow oil (114 mg, 75\%); FT-IR: (MIR-ATR, 4000-600 $\left.\mathrm{cm}^{-1}\right): \mathrm{v}_{\max }=2980.42,1730.22,1550.30,1480.19$, 1295.50, 1220.16, 1193.28, 1040.76, 750.69, 681.90; ${ }^{1} \mathrm{H} \mathrm{NMR}\left(\mathrm{CDCl}_{3}, 400 \mathrm{MHz}\right): \delta_{\mathrm{H}}=7.70(\mathrm{~d}, J=7.34$ $\mathrm{Hz}, 1 \mathrm{H}), 7.31(\mathrm{t}, J=7.83 \mathrm{~Hz}, 2 \mathrm{H}), 7.11(\mathrm{~s}, 2 \mathrm{H}), 6.32(\mathrm{~s}, 2 \mathrm{H}), 4.25(\mathrm{q}, J=7.34 \mathrm{~Hz}, 2 \mathrm{H}), 4.05(\mathrm{q}, J=7.34$ $\mathrm{Hz}, 2 \mathrm{H}), 3.83$ (s, 3H), 3.75 (s, 6H), 1.32 (t, $J=7.09 \mathrm{~Hz}, 3 \mathrm{H}), 1.01$ (t, $J=7.34 \mathrm{~Hz}, 3 \mathrm{H}) ;{ }^{13} \mathrm{C} \mathrm{NMR}\left(\mathrm{CDCl}_{3}\right.$, $100 \mathrm{MHz}): 165.8,164.0,153.6,149.5,144.1,140.1$, 137.5, 135.3, 130.3, 129.2, 127.5, 124.5, 124.1, 101.8, 
87.2, 62.7, 62.1, 61.0, 56.2, 13.9, 13.6; HR-MS (ESI+) $\mathrm{m} / \mathrm{z}$ value calculated for $\left[\mathrm{C}_{23} \mathrm{H}_{26} \mathrm{INaNO}_{4}\right]^{+}=$ $[\mathrm{M}+\mathrm{Na}]^{+}:$578.0650; found: 578.0652.

\section{(Z)Dimethyl 2-((2-fluorophenyl)(phenyl)amino)-3-iodomaleate (4iab)}

Yellow oil; (180 mg, 88\%); FT-IR: (MIR-ATR,4000-600 cm $\left.\mathrm{cm}^{-1}\right): \mathrm{v}_{\max }=2951.5,1990.1,1731.9,1590.7$, 1497.3, 1434.2, 1299.9, 1230.6, 1043.2, 988.9, 757.4, 695.0: ${ }^{1} \mathrm{H} \mathrm{NMR}\left(\mathrm{CDCl}_{3}, 400 \mathrm{MHz}\right): \delta_{\mathrm{H}}=7.27(\mathrm{~m}$, $4 \mathrm{H}) ; 7.12(\mathrm{~m}, 3 \mathrm{H}) ; 6.97(\mathrm{~d}, J=7.8 \mathrm{~Hz}, 2 \mathrm{H}) ; 3.80(\mathrm{~s}, 3 \mathrm{H}) ; 3.58(\mathrm{~s}, 3 \mathrm{H}) ;{ }^{13} \mathrm{C} \mathrm{NMR}\left(\mathrm{CDCl}_{3}, 100 \mathrm{MHz}\right): \delta=$ 166.1, 164.1, 158.9, 156.4, 149.5, 136.4, 129.2, 124.95, 124.43, 121.77, 117.0, 116.8, 86.02, 53.56, 52.94; HR-MS(ESI+) m/z value calculated for $\left[\mathrm{C}_{18} \mathrm{H}_{16} \mathrm{FINO}_{4}\right]^{+}=[\mathrm{M}+\mathrm{H}]^{+}: 456.0103$; found: 456.0096.

\section{(Z)Diethyl 2-((4-chlorophenyl)(phenyl)amino)-3-iodomaleate (4jaa)}

Yellow oil (167 mg, 85\%); FT-IR: (MIR-ATR, 4000-600 $\left.\mathrm{cm}^{-1}\right): \mathrm{v}_{\max }=2980.5,2926.9,2850.7,1725.5$, 1586.9, 1554.1, 1486.6, 1366.3, 1283.9, 1213.8, 1147.2, 1091.3, 1040.8, 823.6, 758.3, 695.5; ${ }^{1} \mathrm{H}$ NMR $\left(\mathrm{CDCl}_{3}, 400 \mathrm{MHz}\right): \delta_{\mathrm{H}}=7.23-7.32(\mathrm{~m}, 4 \mathrm{H}), 7.01-7.14(\mathrm{~m}, 5 \mathrm{H}), 4.22-4.29(\mathrm{q}, J=7.34 \mathrm{~Hz}, 2 \mathrm{H}), 4.03$ $(\mathrm{q}, J=7.34 \mathrm{~Hz}, 2 \mathrm{H}), 1.29-1.34(\mathrm{t}, J=7.09,3 \mathrm{H}), 1.00(\mathrm{t}, J=7.09 \mathrm{~Hz}, 3 \mathrm{H}) ;{ }^{13} \mathrm{C} \mathrm{NMR}\left(\mathrm{CDCl}_{3}, 100 \mathrm{MHz}\right)$ :

$\delta=165.7,163.6,148.6,143.9,142.9,129.5,129.4,124.8,123.8,89.7,62.8,62.2$, 13.9, 13.6; HR-MS (ESI+) $\mathrm{m} / \mathrm{z}$ calculated for $\left[\mathrm{C}_{20} \mathrm{H}_{20} \mathrm{ClINO}_{4}\right]^{+}=[\mathrm{M}+\mathrm{H}]^{+}:$500.0126; found: 500.0139 .

\section{(Z)Diethyl 2-((4-bromophenyl)(phenyl)amino)-3-iodomaleate (4kaa)}

Yellow oil (140 mg, 88\%); FT-IR: (MIR-ATR, 4000-600 $\left.\mathrm{cm}^{-1}\right): \mathrm{V}_{\max }=2890.20,1730.71,1520.35,1461.81$, 1368.26, 1294.03, 1229.20, 1175.10, 1020.22, 754.92, 605.23; ${ }^{1} \mathrm{H} \mathrm{NMR}\left(\mathrm{CDCl}_{3}, 400 \mathrm{MHz}\right): \delta_{\mathrm{H}}=7.36-$ $7.42(\mathrm{~m}, 2 \mathrm{H}), 7.27-7.32(\mathrm{~m}, 2 \mathrm{H}), 7.07-7.14(\mathrm{~m}, 3 \mathrm{H}), 6.94-7.00(\mathrm{~m}, 2 \mathrm{H}), 4.26(\mathrm{q}, J=7.17 \mathrm{~Hz}, 2 \mathrm{H}), 4.04$ $(\mathrm{q}, J=6.85 \mathrm{~Hz}, 2 \mathrm{H}), 1.32(\mathrm{t}, J=7.09 \mathrm{~Hz}, 3 \mathrm{H}), 1.00(\mathrm{t}, J=7.09 \mathrm{~Hz}, 3 \mathrm{H}) ;{ }^{13} \mathrm{C} \mathrm{NMR}\left(\mathrm{CDCl}_{3}, 100 \mathrm{MHz}\right)$ : 165.7, 163.5, 148.4, 143.8, 143.4, 132.3, 129.4, 125.1, 124.8, 123.8, 117.1, 90.2, 62.8, 62.2, 13.9, 13.6; HR-MS (ESI+) m/z value calculated for $\left[\mathrm{C}_{20} \mathrm{H}_{20} \mathrm{BrINO}_{4}\right]^{+}=[\mathrm{M}+\mathrm{H}]^{+}:$547.9650; found: 547.9652 .

\section{(Z)Dimethyl 2-((4-bromophenyl)(phenyl)amino)-3-iodomaleate (4kab)}

Yellow oil (125 mg, 83\%); FT-IR(MIR-ATR, 4000-600 $\left.\mathrm{cm}^{-1}\right): \mathrm{V}_{\max }=2951.6,2923.2,2851.3,1729.9$, $1552.4,1485.4,1433.2,1219.9,1146.6,1043,986.7,816,733,695 ;{ }^{1} \mathrm{H} \mathrm{NMR}\left(\mathrm{CDCl}_{3}, 400 \mathrm{MHz}\right): \delta_{\mathrm{H}}=$ $7.40(\mathrm{~d}, J=8.8 \mathrm{~Hz}, 2 \mathrm{H}) ; 7.31(\mathrm{~m}, 2 \mathrm{H}) ; 7.13(\mathrm{~s}, 1 \mathrm{H}) ; 7.08(\mathrm{~d}, J=7.8 \mathrm{~Hz}, 2 \mathrm{H}) ; 6.96(\mathrm{~d}, J=8.8 \mathrm{~Hz}, 2 \mathrm{H}) ; 3.81$ (s, 3H); 3.59 (s, 3H); ${ }^{13} \mathrm{C} \mathrm{NMR}\left(\mathrm{CDCl}_{3}, 100 \mathrm{MHz}\right): \delta=166.1,164.1,148.6,143.7,143.3,132.4,129.4$, 125.1, 124.9, 123.9, 117.3, 88.9, 53.6, 53.0; HR-MS (ESI+) $\mathrm{m} / \mathrm{z}$ value calculated for $\left[\mathrm{C}_{18} \mathrm{H}_{15} \mathrm{BrINO}_{4}\right]^{+}=$ $[\mathrm{M}+\mathrm{K}]^{+}:$514.9229; found: 514.9225 .

(Z)Diethyl 2-((2-bromophenyl)(phenyl)amino)-3-iodomaleate (4laa) 
Yellow oil (126 mg, 79\%); FT-IR: (MIR-ATR, 4000-600 $\left.\mathrm{cm}^{-1}\right): \mathrm{v}_{\max }=2892.21,1720.74,1527.45,1471.88$, 1368.26, 1294.03, 1219.26, 1176.16, 1026.28, 754.92, 604.33; ${ }^{1} \mathrm{H} \mathrm{NMR}\left(\mathrm{CDCl}_{3}, 400 \mathrm{MHz}\right): \delta_{\mathrm{H}}=7.57$ $7.61(\mathrm{~m}, 1 \mathrm{H}), 7.31-7.37(\mathrm{~m}, 2 \mathrm{H}), 7.21-7.30(\mathrm{~m}, 2 \mathrm{H}), 6.98-7.15(\mathrm{~m}, 3 \mathrm{H}), 6.75$ (br. s., 1H), 4.23 (q, $J=$ $6.85 \mathrm{~Hz}, 2 \mathrm{H}), 4.00(\mathrm{q}, J=6.85 \mathrm{~Hz}, 2 \mathrm{H}), 1.30(\mathrm{t}, J=7.09 \mathrm{~Hz}, 3 \mathrm{H}), 0.97(\mathrm{t}, J=7.09 \mathrm{~Hz}, 3 \mathrm{H}) ;{ }^{13} \mathrm{C} \mathrm{NMR}$ $\left(\mathrm{CDCl}_{3}, 100 \mathrm{MHz}\right):$ 165.7, 163.8, 150.6, 144.3, 142.1, 134.4, 129.8, 129.1, 128.6, 127.7, 124.3, 123.3, 85.0, 62.6, 62.0, 13.9, 13.5; HR-MS (ESI+) $\mathrm{m} / \mathrm{z}$ value calculated for $\left[\mathrm{C}_{20} \mathrm{H}_{20} \mathrm{BrINO}_{4}\right]^{+}=[\mathrm{M}+\mathrm{H}]^{+}: 547.9655$; found: 547.9656

\section{(Z)Diethyl 2-iodo-3-((2-methyl-3-nitrophenyl)(phenyl)amino)maleate (4maa)}

Yellow oil (129 mg, 75\%); FT-IR: (MIR-ATR, 4000-600 cm-1): $\mathrm{v}_{\max }=3018,2949.9,1707,1550.3,1350$, 1288.7, 1199, 1074, 731, 695.2; ${ }^{1} \mathrm{H}$ NMR $\left(\mathrm{CDCl}_{3}, 400 \mathrm{MHz}\right): \delta_{\mathrm{H}}=7.72(\mathrm{~d}, J=7.83 \mathrm{~Hz}, 1 \mathrm{H}), 7.51(\mathrm{~d}, J=$ $7.82 \mathrm{~Hz}, 1 \mathrm{H}), 7.36$ (t, $J=8.07 \mathrm{~Hz}, 1 \mathrm{H}), 7.27$ (s, 2H), $7.08-7.14$ (m, 1H), 7.01 (br. s., 1H), 6.76 (br. s., 1H), $4.24(\mathrm{q}, J=7.34 \mathrm{~Hz}, 2 \mathrm{H}), 4.02(\mathrm{q}, J=7.01 \mathrm{~Hz}, 2 \mathrm{H}), 2.21(\mathrm{~s}, 3 \mathrm{H}), 1.32(\mathrm{t}, J=7.09 \mathrm{~Hz}, 3 \mathrm{H}), 0.99(\mathrm{t}, \quad J=$ $7.09 \mathrm{~Hz}, 3 \mathrm{H}) ;{ }^{13} \mathrm{C} \mathrm{NMR}\left(\mathrm{CDCl}_{3}, 100 \mathrm{MHz}\right): 165.4,163.6,152.0,150.5,144.4,137.5,131.9,130.9,129.5$, 127.3, 126.8, 124.8, 122.0, 85.6, 77.4, 77.1, 76.7, 62.8, 62.3, 15.9, 13.9, 13.5; HR-MS (ESI+) m/z value calculated for $\left[\mathrm{C}_{21} \mathrm{H}_{21} \mathrm{IN}_{2} \mathrm{NaO}_{6}\right]^{+}=[\mathrm{M}+\mathrm{Na}]^{+}:$547.0342; found: 547.0322 .

\section{(Z)Diethyl 2-iodo-3-((4-(methoxycarbonyl)phenyl)(phenyl)amino)maleate (4aba)}

Yellow oil (252 mg, 90\%); FT-IR : (MIR-ATR, 4000-600 $\left.\mathrm{cm}^{-1}\right): \mathrm{v}_{\max }=2982.2,2952.2,1714.8,1590.5$, 1506.7, 1489.8, 1434.6, 1366.6, 1271.9, 1216.4, 1175.6, 1106.7, 1039.5, 767.5, 695.5; ${ }^{1} \mathrm{H} \mathrm{NMR}\left(\mathrm{CDCl}_{3}\right.$, $400 \mathrm{MHz}): \delta_{\mathrm{H}}=7.96(\mathrm{~d}, J=8.8 \mathrm{~Hz}, 2 \mathrm{H}) ; 7.34(\mathrm{~m}, 2 \mathrm{H}) ; 7.17(\mathrm{~m}, 3 \mathrm{H}) ; 7.07(\mathrm{~d}, J=8.3 \mathrm{~Hz}, 2 \mathrm{H}) ; 4.29(\mathrm{q}, J=$ $7.2 \mathrm{~Hz}, 2 \mathrm{H}) ; 4.05(\mathrm{q}, J=6.8 \mathrm{~Hz}, 2 \mathrm{H}) ; 3.89(\mathrm{~s}, 3 \mathrm{H}) ; 1.35(\mathrm{t}, J=7.3 \mathrm{~Hz}, 3 \mathrm{H}) ; 1.01(\mathrm{t}, J=7.3 \mathrm{~Hz}, 3 \mathrm{H}) ;{ }^{13} \mathrm{C}$ NMR ( $\left.\mathrm{CDCl}_{3}, 100 \mathrm{MHz}\right)$ : 166.6, 165.7, 163.1, 148.5, 147.2, 143.1, 131.0, 129.5, 125.5, 124.8, 124.7, 121.2, 93.7, 62.8, 62.3, 52.0, 13.9, 13.6; HR-MS (ESI+) $\mathrm{m} / \mathrm{z}$ value calculated for $\left[\mathrm{C}_{22} \mathrm{H}_{22} \mathrm{INaNO}_{6}\right]^{+}=$ $[\mathrm{M}+\mathrm{Na}]^{+}:$523.0492; found: 523.0488 .

\section{(Z)Dimethyl 2-iodo-3-((4-(methoxycarbonyl)phenyl)(phenyl)amino)maleate (4abb)}

Yellow oil (231 mg, 87\%); FR-IR: (MIR-ATR, 4000-600 cm-1): $\mathrm{v}_{\max }=3001.0,2951.4,2842.4,1713.4$, 1589.6, 1489.4, 1432.9, 1271.4, 1217.8, 1104.8, 1039.7, 800.8, 760.9, 695.6; ${ }^{1} \mathrm{H} \mathrm{NMR}\left(\mathrm{CDCl}_{3}, 400 \mathrm{MHz}\right)$ : $\delta_{\mathrm{H}}=7.96(\mathrm{~d}, J=8.8 \mathrm{~Hz}, 2 \mathrm{H}) ; 7.34(\mathrm{~m}, 2 \mathrm{H}) ; 7.18(\mathrm{~d}, J=8.3 \mathrm{~Hz}, 3 \mathrm{H}) ; 7.05(\mathrm{~d}, J=8.8 \mathrm{~Hz}, 2 \mathrm{H}) ; 3.89(\mathrm{~s}, 3 \mathrm{H})$; 3.84 (s, 3H); 3.6 (s, 3H); ${ }^{13} \mathrm{C} \mathrm{NMR}\left(\mathrm{CDCl}_{3}, 100 \mathrm{MHz}\right): 166.6,166.1,163.8,148.3,147.5,142.9,131.1$, 129.6, 125.6, 124.9, 124.7, 121.2, 92.7, 53.6, 53.0, 52.0; HR-MS (ESI+) m/z value calculated for $\left.\left[\mathrm{C}_{20} \mathrm{H}_{18} \mathrm{IKNO}_{6}\right]^{+}=[\mathrm{M}+\mathrm{K})\right]^{+}\left[\mathrm{H}_{2} 0\right]: 515.9705$; found : 515.9703 .

(Z)Dimethyl 2-iodo-3-((4-(methoxycarbonyl)phenyl)(4-methoxyphenyl)amino)maleate (4gbb) 
Yellow oil (164 mg, 77\%); FT-IR (MIR-ATR, 4000-600 cm-1) $\mathrm{v}_{\max }=2952.1,2924.2,2851.7,1719.9$, 1605.7, 1506.6, 1434.1, 1277.0, 1245.4, 1176.7, 1109.6, 1035.7, 838.2, 769.1, 696.9; ${ }^{1} \mathrm{H} \mathrm{NMR}\left(\mathrm{CDCl}_{3}, 400\right.$ MHz): $\delta_{\mathrm{H}}=7.93(\mathrm{~d}, J=8.8 \mathrm{~Hz}, 2 \mathrm{H}) ; 7.14(\mathrm{~d}, J=8.8 \mathrm{~Hz}, 2 \mathrm{H}) ; 6.96(\mathrm{~d}, J=8.8 \mathrm{~Hz}, 2 \mathrm{H}) ; 6.88(\mathrm{~d}, J=8.8 \mathrm{~Hz}$, $2 \mathrm{H}) ; 3.89$ (s, 3H); 3.82 (s, 3H); 3.61 (s, 3H); ${ }^{13} \mathrm{C} \mathrm{NMR}\left(\mathrm{CDCl}_{3}, 100 \mathrm{MHz}\right)$ : 166.6, 166.0, 164.0, 157.8, 148.8, 148.1, 135.5, 131.0, 127.3, 124.4, 120.0, 114.7, 90.3, 55.5, 53.6, 53.0, 52.0; HR-MS (ESI+) m/z value calculated for $\left[\mathrm{C}_{21} \mathrm{H}_{22} \mathrm{INO}_{7}\right]^{+}=[\mathrm{M}+\mathrm{H}]^{+}:$525.0284; found: 525.0280.

(Z)Dimethyl 2-((4-bromophenyl)(4-(methoxycarbonyl)phenyl)amino)-3-iodomaleate (4kbb)

Yellow oil (152 mg, 91\%); FT-IR (MIR-ATR, 4000-600 $\mathrm{cm}^{-1}$ ) $\mathrm{V}_{\max }=3000.51,2950.90,2838.30,1715.90$, 1595.42, 1498.90, 1423.12, 1270.81, 1222.7, 1094.96, 1049.98, 976.85, 819.89, 769.51, 685.30; ${ }^{1} \mathrm{H}$ NMR $\left(\mathrm{CDCl}_{3}, 400 \mathrm{MHz}\right): \delta_{\mathrm{H}}=7.96(\mathrm{~d}, J=8.80 \mathrm{~Hz}, 2 \mathrm{H}), 7.46(\mathrm{~d}, J=8.80 \mathrm{~Hz}, 2 \mathrm{H}), 7.04(\mathrm{~d}, J=8.31 \mathrm{~Hz}, 4 \mathrm{H})$, 3.90 (s, 3H), 3.84 (s, 3H), 3.62 (s, 3H); ${ }^{13} \mathrm{C}$ NMR $\left(\mathrm{CDCl}_{3}, 100 \mathrm{MHz}\right): 166.5,165.9,163.5,147.9,146.7$, $142.1,132.7,131.8,131.2,126.0,125.3,121.2,118.5,116.2$, 94.1, 53.7, 53.2, 52.1, 29.7; HR-MS (ESI+) $\mathrm{m} / \mathrm{z}$ value calculated for $\left[\mathrm{C}_{20} \mathrm{H}_{17} \mathrm{BrIKNO}_{6}\right]^{+}=[\mathrm{M}+\mathrm{K}]^{+}:$611.8920; found: 611.8921 .

(Z)Diethyl 2-((2-bromo-5-methylphenyl(4-(methoxycarbonyl)phenyl)amino-3-iodomaleate (4nba)

Yellow oil (136 mg, 86\%); FT-IR: (MIR-ATR, 4000-600 $\left.\mathrm{cm}^{-1}\right): \mathrm{v}_{\max }=2981.3,2951.9,1714.3,1606.8$, $1565,1486.9,1434.4,1275,1215.6,1104.8,1014.3,850.3,768.5,735.2,696.5 ;{ }^{1} \mathrm{H} \mathrm{NMR}\left(\mathrm{CDCl}_{3}, 400\right.$ MHz): $\delta_{\mathrm{H}}=7.93(\mathrm{~m}, 2 \mathrm{H}) ; 7.45(\mathrm{~s}, 1 \mathrm{H}) ; 7.24(\mathrm{~s}, 1 \mathrm{H}) ; 7.18(\mathrm{~s}, 1 \mathrm{H}) ; 6.95(\mathrm{br}, \mathrm{s}, 1 \mathrm{H}) ; 6.74$ (br, s, 1H); 4.25 (q, $J=7.3 \mathrm{~Hz}, 2 \mathrm{H}) ; 4.03(\mathrm{q}, J=6.8 \mathrm{~Hz}, 2 \mathrm{H}) ; 3.88(\mathrm{~s}, 3 \mathrm{H}) ; 2.36(\mathrm{~s}, 3 \mathrm{H}) ; 1.32(\mathrm{t}, J=7.1 \mathrm{~Hz}, 3 \mathrm{H}) ; 1.02(\mathrm{t}, J=$ $7.1 \mathrm{~Hz}, 3 \mathrm{H}) ;{ }^{13} \mathrm{C} \mathrm{NMR}\left(\mathrm{CDCl}_{3}, 100 \mathrm{MHz}\right): \delta=166.6,165.6,163.5,149.0,148.4,138.7,138.3,134.8$, 130.8, 129.7, 129.3, 124.8, 123.0, 89.3, 67.1, 62.8, 62.3, 52.0, 20.8, 13.9, 13.6; HR-MS (ESI+) m/z value calculated for $\left[\mathrm{C}_{23} \mathrm{H}_{23} \mathrm{BrINO}_{6}\right]^{+}=[\mathrm{M}+\mathrm{H}]^{+}:$614.9753; found: 614.9749 .

\section{(Z)Diethyl 2-iodo-3-((3-nitrophenyl)(phenyl)amino)maleate (4aca)}

Yellow oil (218 mg, 84\%); FT-IR: (MIR-ATR, 4000-600 $\left.\mathrm{cm}^{-1}\right): \mathrm{v}_{\max }=3006.1,2944.9,1732.9,1558.3$, 1499.7, 1444.1, 1288.7, 1144.4, 1032.8, 968.8, 747.4, 665.4; ${ }^{1} \mathrm{H} \mathrm{NMR}\left(\mathrm{CDCl}_{3}, 400 \mathrm{MHz}\right): \delta_{\mathrm{H}}=7.88-7.93$ (m, 2 H), 7.43 - 7.48 (m, 1 H), 7.33 - 7.39 (m, 3 H), 7.14 - 7.22 (m, 3 H), 4.29 (q, J=7.34 Hz, 2 H), 4.06 (q, $J=7.34 \mathrm{~Hz}, 2 \mathrm{H}), 1.34$ (t, $J=7.09 \mathrm{~Hz}, 3 \mathrm{H}), 1.01$ (t, $J=7.09 \mathrm{~Hz}, 3 \mathrm{H}) ;{ }^{13} \mathrm{C} \mathrm{NMR}\left(\mathrm{CDCl}_{3}, 100 \mathrm{MHz}\right): 165.5$, 163.0, 149.2, 147.2, 145.7, 142.9, 130.1, 129.7, 127.9, 125.7, 124.2, 118.2, 117.0, 93.8, 63.0, 62.4, 13.9, 13.6; HR-MS (ESI+) m/z value calculated for $\left[\mathrm{C}_{20} \mathrm{H}_{19} \mathrm{IN}_{2} \mathrm{NaO}_{6}\right]^{+}=[\mathrm{M}+\mathrm{Na}]^{+}$: 533.0185; found: 533.0188 .

\section{(Z)Dimethyl 2-((4-fluorophenyl)(phenyl)maleate)-3-iodomaleate (4adb)}

Yellow oil (220 mg, 90\%); FT-IR: (MIR-ATR, 400-600 $\left.\mathrm{cm}^{-1}\right): \mathrm{v}_{\max }=2952.9,2916.2,2849.7,1730.8$, 1678.8, 1551.7, 1502.6, 1216.4, 1148.9, 616.8; ${ }^{1} \mathrm{H} \mathrm{NMR}\left(\mathrm{CDCl}_{3}, 400 \mathrm{MHz}\right): \delta_{\mathrm{H}}=7.29(\mathrm{~m}, 2 \mathrm{H}) ; 7.09(\mathrm{~m}$, 
$3 \mathrm{H}) ; 7.02(\mathrm{~m}, 4 \mathrm{H}) ; 3.80(\mathrm{~s}, 3 \mathrm{H}) ; 3.58(\mathrm{~s}, 3 \mathrm{H}) ;{ }^{13} \mathrm{C} \mathrm{NMR}\left(\mathrm{CDCl}_{3}, 100 \mathrm{MHz}\right): \delta=166.1,164.4,161.1,158.7$, 149.6, 144.3 129.4 126.2, 124.6, 123.5, 116.3, 116.1, 86.5, 53.6, 53.9; HR-MS (ESI+) m/z value calculated for $\left[\mathrm{C}_{18} \mathrm{H}_{15} \mathrm{FINO}_{4}\right]^{+}=\left(\mathrm{M}+\mathrm{NH}_{4}\right)^{+}\left[-\mathrm{H}_{2} \mathrm{O}\right]:$ 455.0262; found: 455.0255 .

\section{(Z)Dimethyl 2-((2,4-dimethylphenyl)(4-fluorophenyl)amino)-3-iodomaleate (4oda)}

Yellow oil (173 mg, 87\%); FT-IR: (MIR-ATR, 4000-600 cm-1): $\mathrm{v}_{\max }=2918.74,2850.71,1733.96,1543.47$, 1502.20, 1212.56, 1045.19, 966.76, 828.20, 734.06; ${ }^{1} \mathrm{H} \mathrm{NMR}\left(\mathrm{CDCl}_{3}, 400 \mathrm{MHz}\right): \delta_{\mathrm{H}}=6.79-7.06$ $(\mathrm{m}, 7 \mathrm{H}), 4.20$ (q, $J=6.85 \mathrm{~Hz}, 2 \mathrm{H}), 3.99$ (q, $J=7.34 \mathrm{~Hz}, 2 \mathrm{H}), 2.31$ (s, 3H), 2.06 (s, 3H), 1.29 (t, $J=7.09$ $\mathrm{Hz}, 3 \mathrm{H}), 1.00(\mathrm{t}, J=7.09 \mathrm{~Hz}, 3 \mathrm{H}) ;{ }^{13} \mathrm{C} \mathrm{NMR}\left(\mathrm{CDCl}_{3}, 100 \mathrm{MHz}\right): 165.6,164.2,160.5,158.1,151.9,141.1$, 141.0, 139.4, 136.8, 135.9, 132.3, 128.1, 127.8, 124.9, 124.8, 115.9, 115.7, 79.4, 62.6, 62.0, 21.0, 19.0, 14.0, 13.6; HR-MS (ESI+) m/z value calculated for $\left[\mathrm{C}_{22} \mathrm{H}_{23} \mathrm{FINO}_{4}\right]^{+}=[\mathrm{M}+\mathrm{H}]^{+}:$514.0789; found: 514.0784.

(Z)Diethyl 2-(butyl(phenyl)amino)-3-iodomaleate (4paa)

Yellow oil (216 mg, 71\%); FT-IR (MIR-ATR, 4000-600 cm-1) $\mathrm{V}_{\max }=3002.1,2951.7,1724.9,1610.7$, 1516.6, 1424.1, 1247.0, 1228.4, 1173.7, 1033.7, 832.2, 767.1, 691.9; ${ }^{1} \mathrm{H}$ NMR $\left(\mathrm{CDCl}_{3}, 400 \mathrm{MHz}\right): \delta_{\mathrm{H}}=$ $7.24-7.31(\mathrm{~m}, 2 \mathrm{H}), 6.92-7.00(\mathrm{~m}, 3 \mathrm{H}), 4.21(\mathrm{dq}, J=17.61,7.17 \mathrm{~Hz}, 4 \mathrm{H}), 3.51-3.62(\mathrm{~m}, 2 \mathrm{H}), 1.66-1.76$ $(\mathrm{m}, 2 \mathrm{H}), 1.35-1.41(\mathrm{~m}, 2 \mathrm{H}), 1.31(\mathrm{t}, J=7.09 \mathrm{~Hz}, 3 \mathrm{H}), 1.20(\mathrm{t}, J=7.09 \mathrm{~Hz}, 3 \mathrm{H}), 0.95(\mathrm{t}, J=7.34 \mathrm{~Hz}, 3 \mathrm{H})$; ${ }^{13} \mathrm{C} \mathrm{NMR}\left(\mathrm{CDCl}_{3}, 100 \mathrm{MHz}\right): 165.7,164.2,151.1,145.1,137.5,129.2,122.7,122.3,119.9,85.0,77.4,77.1$, 76.8, 62.5, 62.1, 52.3, 31.1, 20.2, 14.0, 13.9, 13.8; HR-MS (ESI+) $\mathrm{m} / \mathrm{z}$ value calculated for $\left[\mathrm{C}_{18} \mathrm{H}_{24} \mathrm{INNaO}_{4}\right]^{+}=[\mathrm{M}+\mathrm{Na}]^{+}: 468.0648$; found: 468.0644 .

\section{(Z)Diethyl 2-(benzyl(phenyl)amino)-3-iodomaleate (4qaa)}

Yellow oil (168 mg, 75\%); FT-IR (MIR-ATR, 4000-600 $\mathrm{cm}^{-1}$ ) $\mathrm{v}_{\max }=3062.2$, 3030, 2949.92, 1730.9, $1699.3,1621.9,1540.1,1241.4,1030.4,998.0,808.4,730.44,696.07 ;{ }^{1} \mathrm{H} \mathrm{NMR}\left(\mathrm{CDCl}_{3}, 400 \mathrm{MHz}\right): \delta_{\mathrm{H}}=$ $7.42(\mathrm{~d}, J=7.34 \mathrm{~Hz}, 2 \mathrm{H}), 7.33(\mathrm{t}, J=7.58 \mathrm{~Hz}, 2 \mathrm{H}), 7.20-7.26(\mathrm{~m}, 3 \mathrm{H}), 6.93-6.99(\mathrm{~m}, 3 \mathrm{H}), 4.82(\mathrm{~s}, 2 \mathrm{H})$, $4.23(\mathrm{q}, J=6.85 \mathrm{~Hz}, 2 \mathrm{H}), 4.14(\mathrm{q}, J=7.34 \mathrm{~Hz}, 2 \mathrm{H}), 1.31(\mathrm{t}, J=7.09 \mathrm{~Hz}, 3 \mathrm{H}), 1.13(\mathrm{t}, J=7.09 \mathrm{~Hz}, 3 \mathrm{H})$; ${ }^{13} \mathrm{C} \mathrm{NMR}\left(\mathrm{CDCl}_{3}, 100 \mathrm{MHz}\right): 165.7,164.1,151.1,145.5,137.8,129.1,128.5,127.3,127.2,122.6,120.3$, 62.6, 62.2, 56.6, 13.9, 13.7; HR-MS (ESI+) $\mathrm{m} / \mathrm{z}$ value calculated for $\left[\mathrm{C}_{21} \mathrm{H}_{23} \mathrm{INO}_{4}\right]^{+}=[\mathrm{M}+\mathrm{H}]^{+}: 480.0670$; found: 480.0672 .

\section{(Z)Dimethyl 2-(cyclopropyl(phenyl)amino)-3-iodomaleate (4rab)}

Yellow oil (239 mg, 68\%); FT-IR (MIR-ATR, 4000-600 $\mathrm{cm}^{-1}$ ) $\mathrm{v}_{\max }=2988.1,2924.0,1726.4,1645.6$, 1501.6, 1444.1, 1287.4, 1245.4, 1103.6, 1035.7, 818.2, 734.2, 636.3; ${ }^{1} \mathrm{H}$ NMR $\left(\mathrm{CDCl}_{3}, 400 \mathrm{MHz}\right): \delta_{\mathrm{H}}=$ $7.25-7.31(\mathrm{~m}, 2 \mathrm{H}), 7.05$ (d, $J=7.83 \mathrm{~Hz}, 2 \mathrm{H}), 6.95$ (t, $J=7.34 \mathrm{~Hz}, 1 \mathrm{H}), 3.85$ (s, 3H), $3.71(\mathrm{~s}, 3 \mathrm{H}), 2.86$ -

$2.92(\mathrm{~m}, 1 \mathrm{H}), 0.85-0.91$ (m, 4H); ${ }^{13} \mathrm{C} \mathrm{NMR}\left(\mathrm{CDCl}_{3}, 100 \mathrm{MHz}\right): 164.6,163.7,145.0,129.2,121.0,116.2$, 
96.7, 53.5, 53.0, 32.2, 8.6; HR-MS (ESI+) $\mathrm{m} / \mathrm{z}$ value calculated for $\left[\mathrm{C}_{15} \mathrm{H}_{16}\left[\mathrm{KNO}_{4}\right]^{+}=[\mathrm{M}+\mathrm{K}]^{+}: 439.9760\right.$; found: 439.9761.

(Z)Tetraethyl 3,3'-(1,4-phenylenebis(phenylmethylene))bis(2-iodomaleate) (4a”aa)

Yellow oil (212 mg, 54\%); FT-IR (MIR-ATR, 4000-600 cm-1) $\mathrm{v}_{\max }=2952,2853.9,1737.7,1695.7,1536.6$, 1438.1, 1345.4, 1186.7, 1015.7, 828.4, 769.3, 676.2; ${ }^{1} \mathrm{H} \mathrm{NMR}\left(\mathrm{CDCl}_{3}, 400 \mathrm{MHz}\right): \delta_{\mathrm{H}}=7.25-7.33$ $(\mathrm{m}, 6 \mathrm{H}), 7.05-7.10(\mathrm{~m}, 8 \mathrm{H}), 4.25(\mathrm{q}, J=7.01 \mathrm{~Hz}, 4 \mathrm{H}), 4.04(\mathrm{q}, J=6.85 \mathrm{~Hz}, 4 \mathrm{H}), 1.32(\mathrm{t}, J=7.34 \mathrm{~Hz}, 6 \mathrm{H})$, $1.02(\mathrm{t}, J=7.09 \mathrm{~Hz}, 6 \mathrm{H}) ;{ }^{13} \mathrm{C} \mathrm{NMR}\left(\mathrm{CDCl}_{3}, 100 \mathrm{MHz}\right): 165.8,163.8,149.2,144.2,140.7,129.3,125.2$, 124.5, 123.5, 88.2, 62.7, 62.1, 13.9, 13.7; HR-MS (ESI+) $\mathrm{m} / \mathrm{z}$ value calculated for $\left[\mathrm{C}_{34} \mathrm{H}_{35} \mathrm{I}_{2} \mathrm{~N}_{2} \mathrm{O}_{8}\right]^{+}=$ $[\mathrm{M}+\mathrm{H}]^{+}:$853.0480; found: 853.0483.

\section{(Z)Tetramethyl 3,3'-(1,4-phenylenebis(phenylmethylene))bis(2-iodomaleate) (4a”ab)}

Yellow oil (225 mg, 61\%); FT-IR (MIR-ATR, 4000-600 $\mathrm{cm}^{-1}$ ) $\mathrm{v}_{\max }=2953.2,2948,1709,1688,1508.4$, 1495.3, 1434.2, 1214.3, 1183, 1119.4, 1032.7, 752.4, 691.5; ${ }^{1} \mathrm{H}$ NMR $\left(\mathrm{CDCl}_{3}, 400 \mathrm{MHz}\right): \delta_{\mathrm{H}}=7.27-7.33$

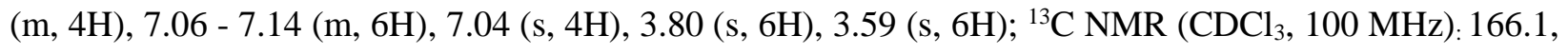
$164.4,149.5,143.9,140.7,129.4,125.2,124.8,123.6,86.9,53.6,53.0$; HR-MS (ESI+) m/z value calculated for $\left[\mathrm{C}_{30} \mathrm{H}_{27} \mathrm{I}_{2} \mathrm{~N}_{2} \mathrm{O}_{8}\right]^{+}=[\mathrm{M}+\mathrm{H}]^{+}:$796.9855; found: 796.9857 .

\section{Diethyl 1-phenyl-1H-indole-2,3-dicarboxylate (5a)}

Yellow liquid (29 mg, 81\%); FT-IR (MIR-ATR, 4000-600 cm-1) $v_{\max }=2950,1737,1707,1539,1501$, $1451,1437,1257,1208,1178,1063,788,755,718 ;{ }^{1} \mathrm{H} \mathrm{NMR}\left(\mathrm{CDCl}_{3}, 400 \mathrm{MHz}\right): \delta_{\mathrm{H}}=8.15(\mathrm{~d}, J=6.85$ $\mathrm{Hz}, 1 \mathrm{H}), 7.41-7.48(\mathrm{~m}, 3 \mathrm{H}), 7.33-7.37(\mathrm{~m}, 2 \mathrm{H}), 7.18-7.26(\mathrm{~m}, 2 \mathrm{H}), 7.10-7.14(\mathrm{~m}, 1 \mathrm{H}), 4.35(\mathrm{q}, J=$ $7.34 \mathrm{~Hz}, 2 \mathrm{H}), 4.16(\mathrm{q}, J=7.01 \mathrm{~Hz}, 2 \mathrm{H}), 1.35(\mathrm{t}, J=7.09 \mathrm{~Hz}, 3 \mathrm{H}), 1.05(\mathrm{t}, J=7.09 \mathrm{~Hz}, 3 \mathrm{H}) ;{ }^{13} \mathrm{C} \mathrm{NMR}$ $\left(\mathrm{CDCl}_{3}, 100 \mathrm{MHz}\right): 164.0,162.4,137.3,136.5,136.3,129.6,129.0,127.3,125.3,124.6,123.0,122.3$, 111.2, 108.3, 62.1, 60.4, 14.4, 13.8; HR-MS (ESI+) $\mathrm{m} / \mathrm{z}$ calculated for $\left[\mathrm{C}_{20} \mathrm{H}_{19} \mathrm{NNaO}_{4}\right]^{+}=[\mathrm{M}+\mathrm{Na}]^{+}$ :360.12063; found: 360.12061 .

\section{Diethyl 1-(o-tolyl)-1H-indole-2,3-dicarboxylate (5b)}

Yellow liquid (28.20 mg, 75\%); FT-IR (MIR-ATR, 4000-600 $\left.\mathrm{cm}^{-1}\right) v_{\max }=2981,1735,1700,1411,1253$, 1181, 1111, 1054, 1010, 737, 610; ${ }^{1} \mathrm{H}$ NMR $\left(\mathrm{CDCl}_{3}, 400 \mathrm{MHz}\right): \delta_{\mathrm{H}}=8.15(\mathrm{~d}, J=7.82 \mathrm{~Hz}, 1 \mathrm{H}), 7.16-7.36$ $(\mathrm{m}, 7 \mathrm{H}), 6.82(\mathrm{~d}, J=8.31 \mathrm{~Hz}, 1 \mathrm{H}), 4.34(\mathrm{q}, J=7.17 \mathrm{~Hz}, 2 \mathrm{H}), 4.10(\mathrm{q}, J=7.34 \mathrm{~Hz}, 2 \mathrm{H}), 1.92(\mathrm{~s}, 3 \mathrm{H}), 1.34$ $(\mathrm{t}, J=7.09 \mathrm{~Hz}, 3 \mathrm{H}), 0.98(\mathrm{t}, J=7.09 \mathrm{~Hz}, 3 \mathrm{H}) ;{ }^{13} \mathrm{C} \mathrm{NMR}\left(\mathrm{CDCl}_{3}, 100 \mathrm{MHz}\right): 164.1,162.1,137.2,137.2$, 136.2, 135.2, 131.0, 129.7, 128.8, 126.9, 125.1, 124.7, 122.9, 122.3, 111.2, 108.1, 61.9, 60.4, 17.3, 14.4, 13.7; HR-MS (ESI+) m/z calculated for $\left[\mathrm{C}_{21} \mathrm{H}_{21} \mathrm{NNaO}_{4}\right]^{+}=[\mathrm{M}+\mathrm{Na}]^{+}: 374.1362$; found: 374.1360 . 


\section{Diethyl 1-(4-bromophenyl)-1H-indole-2,3-dicarboxylate (5c)}

Brown liquid (15 mg, 40\%); FT-IR (MIR-ATR, 4000-600 $\mathrm{cm}^{-1}$ ): $v_{\max }=2981.52,1735.50,1705.87$, $1540.89,1495.40,1451.37,1416.97,1381.75,1255.68,1200.29,1091.59,1041.43,748.16,603.76 ;{ }^{1} \mathrm{H}$ $\operatorname{NMR}\left(\mathrm{CDCl}_{3}, 400 \mathrm{MHz}\right): \delta_{\mathrm{H}}=8.22(\mathrm{~d}, J=7.34 \mathrm{~Hz}, 1 \mathrm{H}), 7.67(\mathrm{~d}, J=7.82 \mathrm{~Hz}, 2 \mathrm{H}), 7.29-7.36(\mathrm{~m}, 4 \mathrm{H})$, $7.17(\mathrm{~d}, J=7.34 \mathrm{~Hz}, 1 \mathrm{H}), 4.42(\mathrm{q}, J=6.85 \mathrm{~Hz}, 2 \mathrm{H}), 4.27(\mathrm{q}, J=6.85 \mathrm{~Hz}, 2 \mathrm{H}), 1.42(\mathrm{t}, J=7.09 \mathrm{~Hz}, 3 \mathrm{H})$, $1.20(\mathrm{t}, J=7.09 \mathrm{~Hz}, 3 \mathrm{H}) ;{ }^{13} \mathrm{C} \mathrm{NMR}\left(\mathrm{CDCl}_{3}, 100 \mathrm{MHz}\right): 163.9,162.2,138.8,137.2,135.8,135.5,132.8$, 129.7, 129.2, 129.1, 128.9, 127.1, 125.3, 124.9, 123.2, 122.9, 122.5, 110.9, 109.0, 62.3, 60.6, 60.5, 14.4, 13.9; HR-MS (ESI+) m/z calculated for $\left[\mathrm{C}_{20} \mathrm{H}_{18} \mathrm{BrNNaO}_{4}\right]^{+}=[\mathrm{M}+\mathrm{Na}]^{+}$: 439.0344; found: 439.0344 .

\section{Diethyl 1-(4-bromophenyl)-1H-indole-2,3-dicarboxylate (5c')}

Yellow liquid (12 mg, 32\%); FT-IR (MIR-ATR, 4000-600 $\left.\mathrm{cm}^{-1}\right) v_{\max }=2981,1702,1443,1409,1251$, 1179, 1061, 694; ${ }^{1} \mathrm{H}$ NMR $\left(\mathrm{CDCl}_{3}, 400 \mathrm{MHz}\right): \delta_{\mathrm{H}}=8.29(\mathrm{~d}, J=1.47 \mathrm{~Hz}, 1 \mathrm{H}), 7.42-7.47(\mathrm{~m}, 3 \mathrm{H}), 7.27-$ $7.34(\mathrm{~m}, 3 \mathrm{H}), 6.98(\mathrm{~d}, J=8.80 \mathrm{~Hz}, 1 \mathrm{H}), 4.34(\mathrm{q}, J=6.85 \mathrm{~Hz}, 2 \mathrm{H}), 4.13-4.18(\mathrm{~m}, 2 \mathrm{H}), 1.34(\mathrm{t}, J=7.09 \mathrm{~Hz}$, 3H), $1.05(\mathrm{t}, J=7.09 \mathrm{~Hz}, 3 \mathrm{H}) ;{ }^{13} \mathrm{C} \mathrm{NMR}\left(\mathrm{CDCl}_{3}, 100 \mathrm{MHz}\right): 163.5,161.9,137.2,136.0,135.9,129.7$, $129.3,127.7,127.1,126.8,124.9,116.6,112.7,107.6,77.4,77.1,76.8,62.3,60.6,14.4,13.8 ;$ HR-MS (ESI+) $\mathrm{m} / \mathrm{z}$ calculated for $\left[\mathrm{C}_{20} \mathrm{H}_{18} \mathrm{BrNNaO}_{4}\right]^{+}=[\mathrm{M}+\mathrm{Na}]^{+}: 440.0291$; found:440.0290.

\section{Diethyl 5-bromo-1-phenyl-1H-indole-2,3-dicarboxylate (5d)}

Brown liquid (26 mg, 66\%); FT-IR (MIR-ATR, 4000-600 $\mathrm{cm}^{-1}$ ): $v_{\max }=2924.22,2852.73,1735.49$, $1703.19,1538.89$, 1505.74, 1451.12, 1411.88, 1379.90, 1255.30, 1182.41, 1150.48, 1110.04, 1054.63, 1012.64, 790.45, 736.98, 611.61; ${ }^{1} \mathrm{H} \mathrm{NMR}\left(\mathrm{CDCl}_{3}, 400 \mathrm{MHz}\right): \delta_{\mathrm{H}}=8.19-8.26(\mathrm{~m}, 1 \mathrm{H}), 7.47-7.55(\mathrm{~m}$, 2H), $7.27-7.41(\mathrm{~m}, 4 \mathrm{H}), 7.16(\mathrm{~d}, J=7.34 \mathrm{~Hz}, 1 \mathrm{H}), 4.42(\mathrm{q}, J=7.17 \mathrm{~Hz}, 2 \mathrm{H}), 4.27(\mathrm{q}, J=7.17 \mathrm{~Hz}, 2 \mathrm{H})$, $1.42(\mathrm{t}, J=7.09 \mathrm{~Hz}, 3 \mathrm{H}), 1.19(\mathrm{t}, J=7.09 \mathrm{~Hz}, 3 \mathrm{H}) ;{ }^{13} \mathrm{C} \mathrm{NMR}\left(\mathrm{CDCl}_{3}, 100 \mathrm{MHz}\right): 163.9,162.2,137.2$, 135.9, 135.0, 135.0, 129.8, 128.6, 125.3, 124.9, 123.2, 122.4, 110.9, 109.0, 62.3, 60.5, 14.4, 13.8; HR-MS $(\mathrm{ESI}+) \mathrm{m} / \mathrm{z}$ calculated for $\left[\mathrm{C}_{22} \mathrm{H}_{23} \mathrm{NNaO}_{4}\right]^{+}=[\mathrm{M}+\mathrm{Na}]^{+}: 388.1519$; found: 388.1516 .

\section{Diethyl 1-(4-chlorophenyl)-1H-indole-2,3-dicarboxylate (5e)}

Yellow liquid (27 mg, 64\%); FT-IR (MIR-ATR, 4000-600 $\left.\mathrm{cm}^{-1}\right) v_{\max }=2949,1704,1538,1477,1436,1250$, 1206, 1174, 1074, 1073, 748; ${ }^{1} \mathrm{H}$ NMR $\left(\mathrm{CDCl}_{3}, 400 \mathrm{MHz}\right): \delta_{\mathrm{H}}=8.15-8.20(\mathrm{~m}, 1 \mathrm{H}), 7.75-7.79(\mathrm{~m}, 1 \mathrm{H})$, $7.39-7.51(\mathrm{~m}, 3 \mathrm{H}), 7.29-7.36(\mathrm{~m}, 2 \mathrm{H}), 6.93(\mathrm{~d}, J=7.34 \mathrm{~Hz}, 1 \mathrm{H}), 3.97(\mathrm{~s}, 3 \mathrm{H}), 3.77(\mathrm{~s}, 3 \mathrm{H}) ;{ }^{13} \mathrm{C} \mathrm{NMR}$ $\left(\mathrm{CDCl}_{3}, 100 \mathrm{MHz}\right): 164.5,162.0,137.3,136.0,134.2,133.7,131.0,130.5,128.4,125.3,125.1,123.3$, 
123.1, 122.4, 111.4, 110.2, 52.8, 51.8; HR-MS (ESI+) $\mathrm{m} / \mathrm{z}$ calculated for $\left[\mathrm{C}_{18} \mathrm{H}_{14} \mathrm{BrNNaO}_{4}\right]^{+}=[\mathrm{M}+\mathrm{Na}]^{+}$: 411.9978; found: 411.9990 .

\section{Diethyl 1-(4-methoxyphenyl)-1H-indole-2,3-dicarboxylate (5f \& 5f')}

Yellow liquid (28 mg, 72\%); FT-IR (MIR-ATR, 4000-600 $\left.\mathrm{cm}^{-1}\right) v_{\max }=2980,1868,1827,1482,1454,1411$, 1252, 1209, 1159, 1117, 1058, 102, 758, 697; ${ }^{1} \mathrm{H}$ NMR $\left(\mathrm{CDCl}_{3}, 400 \mathrm{MHz}\right): \delta_{\mathrm{H}}=8.19-8.24$ (m, 1H), 7.69 (d, J = 1.96 Hz, 1H), $7.47-7.55$ (m, 3H), $7.39-7.43(\mathrm{~m}, 2 \mathrm{H}), 7.25$ - 7.36 (m, 4H), 7.15 (d, $J$ $=7.83 \mathrm{~Hz}, 1 \mathrm{H}), 7.09(\mathrm{~d}, J=8.80 \mathrm{~Hz}, 1 \mathrm{H}), 7.00-7.04(\mathrm{~m}, 2 \mathrm{H}), 6.93(\mathrm{dd}, J=9.05,2.69 \mathrm{~Hz}, 1 \mathrm{H}), 4.41(\mathrm{qd}$, $J=7.17,2.93 \mathrm{~Hz}, 4 \mathrm{H}), 4.24(\mathrm{dq}, J=11.31,7.15 \mathrm{~Hz}, 4 \mathrm{H}), 3.91$ (s, 3H), 3.88 (s, 2H), 1.41 (td, $J=7.09,3.42$ $\mathrm{Hz}, 6 \mathrm{H}), 1.17$ (t, $J=7.09 \mathrm{~Hz}, 3 \mathrm{H}), 1.13(\mathrm{t}, J=7.09 \mathrm{~Hz}, 3 \mathrm{H}) ;{ }^{13} \mathrm{C} \mathrm{NMR}\left(\mathrm{CDCl}_{3}, 100 \mathrm{MHz}\right): 164.2,164.1$, $162.3,159.9,156.5,137.7,136.6,136.2,132.4,129.5,129.0,128.9,128.6,127.1,126.2,125.4,125.2$, 124.5, 122.9, 122.2, 115.5, 114.6, 112.1, 111.2, 107.8, 103.0, 62.1, 62.1, 60.3, 60.3, 55.7, 55.6, 14.4, 14.4, 13.9, 13.8; HR-MS (ESI+) m/z calculated for $\left[\mathrm{C}_{21} \mathrm{H}_{21} \mathrm{NNaO}_{5}\right]^{+}=[\mathrm{M}+\mathrm{Na}]^{+}: 390.1312$; found: 390.1314 .

\section{Diethyl 1-(2,4-dimethylphenyl)-1H-indole-2,3-dicarboxylate (5g)}

Yellow semisolid (30.46 mg, 78\%); IR (MIR-ATR, 4000-600 cm-1) $v_{\max }=2981,1735,1700,1411,1253$, 1181, 1111, 1054, 1010,737,610; ${ }^{1} \mathrm{H}$ NMR $\left(\mathrm{CDCl}_{3}, 400 \mathrm{MHz}\right): \delta_{\mathrm{H}}=8.15$ (d, J=7.82 Hz, 1H), $7.16-7.26$ $(\mathrm{m}, 2 \mathrm{H}), 7.01-7.13(\mathrm{~m}, 3 \mathrm{H}), 6.83(\mathrm{~d}, J=7.83 \mathrm{~Hz}, 1 \mathrm{H}), 4.31-4.38(\mathrm{~m}, 2 \mathrm{H}), 4.12(\mathrm{q}, J=7.17 \mathrm{~Hz}, 2 \mathrm{H}), 2.34$ $(\mathrm{s}, 3 \mathrm{H}), 1.88(\mathrm{~s}, 3 \mathrm{H}), 1.35$ (t, $J=7.09 \mathrm{~Hz}, 3 \mathrm{H}), 1.03(\mathrm{t}, J=7.34 \mathrm{~Hz}, 3 \mathrm{H}) ;{ }^{13} \mathrm{C} \mathrm{NMR}\left(\mathrm{CDCl}_{3}, 100 \mathrm{MHz}\right)$ : $164.2,162.2$, 139.7, 137.3, 136.8, 136.3, 132.5, 131.7, 128.5, 127.5, 125.1, 124.5, 122.8, 122.3, 111.2, $107.8,61.9,60.3,21.3,17.2,14.4,13.7$; HR-MS (ESI+) $\mathrm{m} / \mathrm{z}$ calculated for $\left[\mathrm{C}_{22} \mathrm{H}_{23} \mathrm{NNaO}_{4}\right]^{+}=[\mathrm{M}+\mathrm{Na}]^{+}$: 388.1519; found: 388.1535 .

\section{Diethyl 1-(3-methyl-2-nitrophenyl)-1H-indole-2,3-dicarboxylate (5h)}

Yellow liquid (28.8 mg, 68\%); IR (MIR-ATR, 4000-600 $\left.\mathrm{cm}^{-1}\right) v_{\max }=2983,1704,1531,1350,1261,1199$, 1199, 1174, 1074, 731; ${ }^{1} \mathrm{H}$ NMR $\left(\mathrm{CDCl}_{3}, 400 \mathrm{MHz}\right): \delta_{\mathrm{H}}=8.14(\mathrm{~d}, J=6.85 \mathrm{~Hz}, 1 \mathrm{H}), 7.98(\mathrm{dd}, J=7.83$, $0.98 \mathrm{~Hz}, 1 \mathrm{H}), 7.40-7.51(\mathrm{~m}, 2 \mathrm{H}), 7.20-7.32(\mathrm{~m}, 2 \mathrm{H}), 6.80(\mathrm{~d}, J=7.82 \mathrm{~Hz}, 1 \mathrm{H}), 4.37$ (q, J= 7.34 Hz, 2H), $4.14(\mathrm{q}, J=7.09,1.22 \mathrm{~Hz}, 2 \mathrm{H}), 2.06(\mathrm{~s}, 3 \mathrm{H}), 1.36(\mathrm{t}, J=7.09 \mathrm{~Hz}, 3 \mathrm{H}), 1.05(\mathrm{t}, J=7.09 \mathrm{~Hz}, 3 \mathrm{H}) ;{ }^{13} \mathrm{C}$ NMR $\left(\mathrm{CDCl}_{3}, 100 \mathrm{MHz}\right): 163.8,161.4,151.0,137.7,137.2,134.8,133.5,133.0,127.2$, 125.6, 125.2, 123.4, 122.7, 110.8, 110.2, 77.4, 77.1, 76.8, 62.1, 60.7, 14.4, 14.1, 13.8; HR-MS (ESI+) m/z calculated for $\left[\mathrm{C}_{21} \mathrm{H}_{20} \mathrm{~N}_{2} \mathrm{NaO}_{6}\right]^{+}=[\mathrm{M}+\mathrm{Na}]^{+}: 419.1214$; found: 419.1219 . 


\section{References:}

1. For difunctionalization of alkynes see: (a) Controlled Trifluoromethylation Reactions of Alkynes through Visible-Light Photoredox Catalysis. Iqbal, N.; Jung, J.; Park, S.; Cho, E. J. Angew. Chem., Int. Ed. 2014, 53, 539-542. (b) Electrophilic Trifluoromethylation by Copper-Catalyzed Addition of $\mathrm{CF}_{3}$-Transfer Reagents to Alkenes and Alkynes. Janson, P. G.; Ghoneim, I.; Ilchenko, N. O.; Szabo, K. J. Org. Lett. 2012, 14, 2882-2885. (c) Generation of benzosultams viatrifluoromethylation of 2-ethynylbenzenesulfonamide under visible light. Xiang, Y.; Kuang, Y.; Wu, J. Org. Chem. Front. 2016, 3, 901-905. (d) A new strategy to construct a C=C$\mathrm{CF}_{3}$ subunit via $\mathrm{CuBr}$-catalyzed domino reaction of homopropargyl amines: an efficient synthesis of trifluoromethyl containing building blocks 4-trifluoromethyl-2,3-dihydro-pyrroliums. Ge, G.C.; Huang, X.-J.; Ding, C.-H.; Wan, S.-L.; Dai, L.-X.; Hou, X.-L. Chem. Commun. 2014, 50, 30483051. (e) A new strategy to construct a $\mathrm{C}=\mathrm{C}-\mathrm{CF}_{3}$ subunit via $\mathrm{CuBr}$-catalyzed domino reaction of homopropargyl amines: an efficient synthesis of trifluoromethyl containing building blocks 4trifluoromethyl-2,3-dihydro-pyrroliums. Lv, Y.; Pu, W.; Chen, Q.; Wang, Q.; Niu, J.; Zhang, Q. J. Org. Chem. 2017, 82, 8282-8289. (f) Copper-Catalyzed Hydroxyl-Directed Aminoarylation of Alkynes. Sun, J.; Zheng, G.; Xiong, T.; Zhang, Q.; Zhao, J.; Li, Y.; Zhang, Q. ACS Catal. 2016, 6, 3674-3678. (g) Radical cascade reaction of alkynes with $N$-fluoroarylsulfonimides and alcohols. Zheng, G.; Li, Y.; Han, J.; Xiong, T.; Zhang, Q. Nat. Commun. 2015, 6, 7011.

2. (a) Regioselective Iodoazidation of Alkynes: Synthesis of $\alpha, \alpha$-Diazidoketones. Okamoto, N.; Sueda, T.; Minami, H.; Miwa, Y.; Yanada, R. Org. Lett. 2015, 17, 1336-1339. (b) CopperCatalyzed Azidative Multifunctionalization of Alkynes. Zheng, G.; Sun, J.; Liu, Y.; Yang, S.; Li, Y.; Sun, H.; Zhang. Q. J. Org. Chem. 2017, 82, 12813-12820.

3. (a) Iodine-promoted cyclization of $N$-propynyl amides and $N$-allyl amides via sulfonylation and sulfenylation. Senadi, G.; C.; Guo, B.-C.; Ju, W.-P.; Wang, J.-J. Chem. Commun. 2016, 52, 1141011413. (b) Metal-free selenosulfonylation of alkynes: rapid access to $\beta$-(seleno)vinyl sulfones via a 
cationic-species-induced pathway. Sun, K.; Wang, X.; Fu, F.; Zhang, C.; Chen, Y.; Liu, L.; Green Chem. 2017, 19, 1490-1493.

4. (a) $\mathrm{PhI}(\mathrm{OAc})_{2}$-mediated 1,2-aminohalogenation of alkynes: a general access to (E)-4(halomethylene)oxazolidin-2-ones. Wang, R.; Zou, H.; Xiong, Y.; Yi, N.; Deng, W.; Xiang, J. Org. Biomol. Chem. 2017, 15, 3964-3967. (b) A General $\mathrm{CuCl}_{2}$-Promoted Alkene Aminochlorination Reaction. Li, S.; Xiong, P.; Zhu, L.; Qian, X.; Xu, H.; Eur. J. Org. Chem. 2016, 3449-3455.

5. Stereodivergent Silylzincation of $\alpha$-Heteroatom-Substituted Alkynes. Fopp, C.; Romain, E.; Isaac, K.; Chemla, F.; Ferreira, F.; Jackowski, O.; Oestreich, M.; Perez-Luna, A. Org. Lett. 2016, 18, 2054-2057.

6. Regio- and Stereoselective Iodoacyloxylations of Alkynes. Priebbenow, D. L.; Gable, R. W.; Baell, J. J. Org. Chem. 2015, 80, 4412-4418.

7. (a) Regio- and Stereoselective Radical Perfluoroalkyltriflation of Alkynes Using Phenyl(perfluoroalkyl)iodonium Triflates. Wang, X.; Studer, A. Org. Lett. 2017, 19, 2977-2980. (b) Pd-Catalyzed Stereoselective Carboperfluoroalkylation of Alkynes. Li, Z.; García-Domínguez, A.;_Nevado, C. J. Am. Chem. Soc. 2015, 137, 11610-11613.

8. (a) Synthesis of Heterocycles from Alkyl 3-(Dimethylamino)propenoates and Related Enaminones. Stanovnik, B.; Svete, J. Chem. Rev. 2004, 104, 2433-2480. (b) Synthesis of N-substituted carbazolones from $\alpha$-iodo enaminones via $\mathrm{Pd}(0)$-catalyzed intramolecular coupling under microwave irradiation. Yun, X-L.; Bi, W-Y.; Huang, J-H.; Liu, Y.; Zhang-Negrerie, D.; Du, Y-F.; Zhao K. Tetrahedron Lett. 2012, 53, 5076-5080. (c) Visible-Light-Induced Photocatalytic Reductive Transformations of Organohalides. Kim, E.; Lee, C. Angew. Chem. Int. Ed. 2012, 51, 12303. (d) Intramolecular 1,5-H transfer reaction of aryl iodides through visible-light photoredox catalysis: a concise method for the synthesis of natural product scaffolds. Chen, J. Q.; Wei, Y. L.; Xu, G. Q.; Liang, Y. M.; Xu, P. F. Chem. Commun. 2016, 52, 6455.

9. Concurrent $\alpha$-Iodination and N-Arylation of Cyclic $\beta$-Enaminones. Chen, Y.; Ju, T.; Wang, J.; Yu, W.; Du, Y.; Zhao, K. Synlett 2010, 2, 231-234. 
10. $\mathrm{PhI}(\mathrm{OAc})_{2}$ mediated 1,2-aminohalogenation of alkynes: a general access to (E)-4-(halomethylene) oxazolidin-2-ones. Wang, R.; Zou, H.; Xiong, Y.; Yi, N.; Deng, W.; Xiang, J. Org. Biomol. Chem. 2017, 15, 3964-3967.

11. Palladium-Catalyzed Intermolecular Dehydrogenative Aminohalogenation of Alkenes under Molecular Oxygen: An Approach to Brominated Enamines. Ji, X.; Huang, H.; Wu, W.; Jiang, H. J. Am. Chem. Soc. 2013, 135, 5286-5289.

12. Stereoselective Chlorination and Bromination of Enamides and Enamines via an Electrostatic Attraction Effect Using (1,1-Diacetoxyiodo)benzene and a Halide Source. Xing, L.; Li, C. J. Org. Chem. 2015, 80, 10000-10008.

13. (a) Alkyne aminohalogenation enabled by DBU-activated N-haloimides: direct synthesis of halogenated enamines. Li, M.; Yuan, H.; Zhao, B.; Liang, F.; Zhang J. Chem. Commun. 2014, 50, 2360-2363. (b) A Catalytic Reaction of Alkynes via Multiple-Site Functionalization. Karur, S.; Kotti, S. R. S. S.; Xu, X.; Cannon, J. F.; Headley, A.; Li, G. J. Am. Chem. Soc. 2003, 125, 1334013341.

14. (a) Arepally, S.; Babu, V. N.; Bakhadoss, M.; Sharada, D. S. A Direct Cycloaminative Approach to Imidazole Derivatives via Dual C-H Functionalization. Org Lett. 2017, 19, 5014-5017. (b) Sagar, A.; Vidyacharan, S.; Shinde, A. H.; Sharada, D. S. Hypervalent iodine(III)-promoted Nincorporation into $\mathrm{N}$-aryl vinylogous carbamates to quinoxaline diesters: access to $1,4,5,8$ tetraazaphenanthrene. Org. Biomol. Chem. 2016, 14, 4018-4022.

15. (a) Hypervalent iodine(III)-induced oxidative [4+2] annulation of $o$-phenylenediamines and electron-deficient alkynes: direct synthesis of quinoxalines from alkyne substrates under metal-free conditions. Okumura, S.; Takeda, Y.; Kiyokawa K.; Minakata, S. Chem. Commun. 2013, 49, 92669268. (b) PIDA-Mediated Oxidative C-C Bond Formation: Novel Synthesis of Indoles from NAryl Enamines Yu, W.; Du, Y.; Zhao K. Org. Lett. 2009, 11, 2417-2420. (c) Synthesis of Chromeno[2,3-b]indol-11(6H)-one via $\mathrm{PhI}(\mathrm{OAc})_{2}$-Mediated Intramolecular Oxidative $\mathrm{C}\left(\mathrm{sp}^{2}\right)$ $\mathrm{N}\left(\mathrm{H}_{2}\right)$ Bond Formation. Sun, J.; Zhang-Negrerie, D.; Du, Y.; Zhao, K. J. Org. Chem. 2015, 80, 
1200-1206. (d) Hypervalent Iodine-Mediated Intramolecular trans-Aminocarboxylation and Oxoaminocarboxylation of Alkynes: Divergent Cascade Annulations of Isocoumarins under MetalFree Conditions. Zhang, X.; Hou, W.; Zhang-Negrerie, D.; Zhao, K.; Du, Y. Org. Lett. 2015, 17, 5252-5255. (e) Hypervalent Iodine-Mediated Oxygenation of N,N-Diaryl Tertiary Amines: Intramolecular Functionalization of $\mathrm{sp}^{3} \mathrm{C}-\mathrm{H}$ Bonds Adjacent to Nitrogen. Zhang, N.; Cheng, R.; Zhang-Negrerie, D.; Du, Y.; Zhao, K. J. Org. Chem. 2014, 79,10581-10587.

16. (a) Hypervalent Iodine(III) in Direct Oxidative Amination of Arenes with Heteroaromatic Amines, Manna, S.; Serebrennikova, P.O.; Utepova, I.A.; Antonchick, A.P.; Chupakhin, O.N. Org. Lett. 2015, 17, 4588-4591. (b) Hypervalent Iodine(III) in Direct Carbon-Hydrogen Bond Functionalization, Narayan, R.; Manna, S.; Antonchick, A. P. Synlett 2015, 26, 1785-1803.

17. (a) Palladium(II)-Catalyzed Direct Arylation of Enaminones Using Organotrifluoroborates. Ge, H.; Niphakis, M. J.; Georg, G. I. J. Am. Chem. Soc. 2008, 130, 3708-3709. (b) Palladium-catalyzed direct $\mathrm{C}-\mathrm{H}$ arylation of enamides with simple arenes. Pankajakshan, S.; Xu, Y.-H.; Cheng, J. K.; Low, M. T.; Loh, T.-P. Angew. Chem., Int. Ed. 2012, 51, 5701. (c) Microwave-assisted SuzukiMiyaura couplings on $\alpha$-iodoenaminones. Wang, X.; Turunen, B. J.; Leighty, M. W.; Georg, G. I. Tetrahedron Lett. 2007, 48, 8811-8814. (d) Stereoselective syntheses of piperidinones and their modification by organometallic coupling reactions. Kranke, B.; Kunz, H. Org. Biomol. Chem. 2007, 5, 349-354. (e) Regiospecific substitution of $N$-acyl-2,3-dihydro-4-pyridones at C-5 via halogenation and cross-coupling. Comins, D. I.; Joseph, S. P.; Chen, X. Tetrahedron Lett. 1995, 36, 9141-9144.

18. Palladium-Catalyzed Oxidative Cyclization of Tertiary Enamines for Synthesis of 1,3,4Trisubstituted Pyrroles and 1,3-Disubstituted Indoles. Lian, X.-L.; Ren, Z.-H.; Wang, Y.-Y.; Guan, Z.-H. Org. Lett. 2014, 16, 3360-3363.

19. Simple and Practical Method for Preparation of [(Diacetoxy)iodo]arenes with Iodoarenes and $m$ Chloroperoxybenzoic Acid. Iinuma, M.; Moriyama, K.; Togo, H. Synlett 2012, 23, 2663-2666. 